\title{
The Power of Imaging: Constraining the Plasma Properties of GRMHD Simulations Using EHY Observations of Sgr A*
}

\section{Citation}

Chan, Chi-Kwan, Dimitrios Psaltis, Feryal Özel, Ramesh Narayan, and Aleksander Sa, dowski. 2015. "The Power of Imaging: Constraining the Plasma Properties of GRMHD Simulations Using EHY Observations of Sgr A*" The Astrophysical Journal 799 (1) (January 9): 1. doi:10.1088/0004-637x/799/1/1.

\section{Published Version}

doi:10.1088/0004-637X/799/1/1

\section{Permanent link}

http://nrs.harvard.edu/urn-3:HUL.InstRepos:27801311

\section{Terms of Use}

This article was downloaded from Harvard University's DASH repository, and is made available under the terms and conditions applicable to Other Posted Material, as set forth at http:// nrs.harvard.edu/urn-3:HUL.InstRepos:dash.current.terms-of-use\#LAA

\section{Share Your Story}

The Harvard community has made this article openly available.

Please share how this access benefits you. Submit a story.

Accessibility 


\title{
THE POWER OF IMAGING: CONSTRAINING THE PLASMA PROPERTIES OF GRMHD SIMULATIONS USING EHT OBSERVATIONS OF Sgr A*
}

\author{
Chi-Kwan Chan $^{1}$, Dimitrios Psaltis ${ }^{1}$, Feryal Özel ${ }^{1}$, Ramesh Narayan $^{2}$, and Aleksander Sadowski ${ }^{3}$ \\ ${ }^{1}$ Steward Observatory and Department of Astronomy, University of Arizona, 933 N. Cherry Ave., Tucson, AZ 85721, USA; chanc@email.arizona.edu \\ ${ }^{2}$ Institute for Theory and Computation, Harvard-Smithsonian Center for Astrophysics, 60 Garden Street, Cambridge, MA 02138, USA \\ ${ }^{3}$ MIT Kavli Institute for Astrophysics and Space Research, 77 Massachusetts Ave., Cambridge, MA 02139, USA \\ Received 2014 October 9; accepted 2014 November 1; published 2015 January 9
}

\begin{abstract}
Recent advances in general relativistic magnetohydrodynamic simulations have expanded and improved our understanding of the dynamics of black-hole accretion disks. However, current simulations do not capture the thermodynamics of electrons in the low density accreting plasma. This poses a significant challenge in predicting accretion flow images and spectra from first principles. Because of this, simplified emission models have often been used, with widely different configurations (e.g., disk- versus jet-dominated emission), and were able to account for the observed spectral properties of accreting black holes. Exploring the large parameter space introduced by such models, however, requires significant computational power that exceeds conventional computational facilities. In this paper, we use GRay, a fast graphics processing unit (GPU) based ray-tracing algorithm, on the GPU cluster El Gato, to compute images and spectra for a set of six general relativistic magnetohydrodynamic simulations with different magnetic field configurations and black-hole spins. We also employ two different parametric models for the plasma thermodynamics in each of the simulations. We show that, if only the spectral properties of Sgr A* are used, all 12 models tested here can fit the spectra equally well. However, when combined with the measurement of the image size of the emission using the Event Horizon Telescope, current observations rule out all models with strong funnel emission, because the funnels are typically very extended. Our study shows that images of accretion flows with horizon-scale resolution offer a powerful tool in understanding accretion flows around black holes and their thermodynamic properties.
\end{abstract}

Key words: accretion, accretion disks - black hole physics - Galaxy: center - radiative transfer

\section{INTRODUCTION}

General relativistic magnetohydrodynamic (GRMHD) simulations of accretion flows onto black holes have significantly expanded and improved our understanding of accretion physics (see Abramowicz \& Fragile 2013; Yuan \& Narayan 2014 for recent reviews). Multiple numerical algorithms have been developed, which allow for accurate simulations of the turbulent magnetohydrodynamic (MHD) flow in the curved spacetime near a black hole, the plunging of matter at the location of the innermost stable circular orbit, the accretion through the event horizon, and the generation of outflows and jets.

An important limitation of all current GRMHD simulations is their inability to follow the thermodynamic properties of the electrons in the flow. This is a crucial ingredient in predicting the observational characteristics of accretion flows onto blackholes, because the emission from such systems is dominated by the radiative properties of the electrons. There are several reasons that hamper rapid progress in this aspect of global simulations. First, the heating and, in general, the acceleration of electrons by the dissipation of the MHD turbulence occurs at sub-grid scales (see, e.g., Riquelme et al. 2012). Second, the transport of heat by conduction is highly anisotropic and takes place in a regime that is poorly understood (see, e.g., Sharma et al. 2008). Finally, radiative energy losses are characterized by cross sections that have very strong dependence on photon energy and render approximate radiative transfer schemes inadequate (see, e.g., Davis et al. 2012).

Improving our understanding of the relevant physical process will be stimulated in the near future by technological advances in spatially resolved imaging observations of black holes. The
Event Horizon Telescope (EHT) will perform millimeter very long baseline interferometry (VLBI) observations of a number of supermassive black holes and will achieve horizon-scale resolution for at least two well studied systems, Sgr A* and M87. Early EHT observations have confirmed that the size of the emitting region at $1.3 \mathrm{~mm}$ in both systems is at most equal to a few Schwarzschild radii (Doeleman et al. 2008, 2012). This simple piece of evidence, in combination with the broadband spectra of the sources, can already place stringent constraints on the physical conditions and geometries of the accretion flows (see, e.g., Broderick et al. 2009, 2011a; Dexter et al. 2009, 2010, 2012; Dexter \& Fragile 2011; Shcherbakov et al. 2012; Psaltis et al. 2014). In the near future, incorporating ALMA and the South Pole Telescope to the array of telescopes that comprise the EHT will generate polarization dependent images of Sgr $\mathrm{A}^{*}$ and M87 at two wavelengths $(1.3 \mathrm{~mm}$ and $0.8 \mathrm{~mm})$ and at different epochs (see, e.g., Doeleman et al. 2009; Broderick et al. 2011b). This wealth of data will provide the observational foundation against which the results of GRMHD simulations will be calibrated.

The power of comparing theoretical models to spatially resolved observations of accreting black holes becomes apparent when exploring the relative importance of the shearing flow (or, for simplicity, the disk) and of the bulk outflow or jet in a system. Albeit typically absent from simple analytic models of accretion disks, outflows, such as highly relativistic jets and winds are common features of GRMHD simulations that often form spontaneously (see, e.g., McKinney \& Gammie 2004). The plasma density in an outflow is typically much lower than in the disk but the magnetic field and relativistic Lorentz factor are much larger, potentially dominating the emerging radiation 
from the system. Indeed, semi-analytic models dominated by a radiatively inefficient accretion flow (see, e.g., Narayan et al. 1998) or by a relativistic jet (see, e.g., Falcke \& Markoff 2000) have both been used to explain the spectra and inferred image sizes of Sgr A*. Such conceptually different geometries will be readily distinguishable using the combination of horizon-scale resolution, sensitivity to different polarizations, and ability to follow the variability of emission that will become available with the complete EHT array.

Our aim is to investigate the ability of future EHT observations to distinguish between different emission geometries, black-hole properties, and thermodynamic conditions in the accreting plasma around Sgr $\mathrm{A}^{*}$. In this first paper, we explore a large suite of GRMHD simulations onto black holes (Narayan et al. 2012; Sądowski et al. 2013a) with different black-hole spins, different prescriptions regarding the thermodynamic properties of the electrons, and different magnetic field topologies of the saturated state of the turbulent flow. Among the very large range of possible configurations, we select those that agree with the broadband spectrum of Sgr A* as well as with the initial measurement of the size of its image at $1.3 \mathrm{~mm}$. In follow-up articles, we will use this suite of models to make detailed predictions for EHT observations and to develop observing strategies that will maximize the scientific return of the EHT.

In earlier work, GRMHD simulations have been used to explore the effect of changing the black-hole spin magnitude and orientation with respect to the observer (Dexter et al. 2010; Mościbrodzka et al. 2009), the tilt of the black-hole spin with respect to the angular momentum of the accreting flow (Dexter \& Fragile 2013), and the thermodynamic properties of the electrons in the outflow/jet (Mościbrodzka \& Falcke 2013; Mościbrodzka et al. 2014). Our work improves on three aspects of these earlier studies. First, we consider the influence of the large-scale magnetic field in the saturated state of the flow, by contrasting the simulations with Magnetically Arrested Disks (MAD) to those with Standard And Normal Evolution (SANE) of Narayan et al. (2012) and Sądowski et al. (2013a). These two types of simulations have very different dynamical behaviors, as well as very different magnetic field topologies near the blackhole horizon. Second, the GRMHD simulations that we are using have been evolved long enough for the flow to reach a dynamical steady state out to about a hundred gravitational radii. This is important because a very large volume of the accretion flow contributes to the low-frequency synchrotron emission as well as to the X-ray bremsstrahlung emission. Finally, owing to our use of a very fast radiative transport algorithm, instead of calculating the time-averaged properties of the simulated flows before constructing images and spectra like in many previous studies, we are able to calculate images and spectra for each snapshot of the flow, before averaging them together. This approach was also taken by Dexter et al. $(2009,2010)$ and Dexter \& Fragile (2013). Albeit computationally very expensive, it mimics more closely the averaging that will inevitably occur during the EHT observations and produces results that differ considerably from the alternate procedure.

In order to achieve the high efficiency of ray tracing required to calculate images and broadband spectra of every snapshot of a GRMHD simulation, we employ our algorithm GRay (Chan et al. 2013). Unlike standard central processing unit (CPU)-based ray tracing algorithms, GRay uses graphics processing units (GPUs) to accelerate the computationally intensive geodesic integration. With careful handling of the access to the memory,
Table 1

Summary of the Six Sets of GRMHD Models of Black-hole Accretion Systems used in this Study; their Detailed Descriptions can be Found in Narayan et al. (2012) and Sa̧dowski et al. (2013a)

\begin{tabular}{lcccc}
\hline Model & $\begin{array}{c}\text { Black Hole } \\
\text { Spin } a\end{array}$ & $\begin{array}{c}\text { Initial } \\
B \text { Field }\end{array}$ & $\begin{array}{c}\text { Resolution } \\
(r, \phi, \theta)\end{array}$ & $\begin{array}{c}\text { Snapshots Used } \\
\text { in } G M c^{-3}\end{array}$ \\
\hline aOSANE & 0.0 & Multi-loop & $256 \times 128 \times 64$ & $230,000-230,990$ \\
a7SANE & 0.7 & Multi-loop & $256 \times 128 \times 64$ & $103,000-103,990$ \\
a9SANE & 0.9 & Multi-loop & $256 \times 128 \times 64$ & $54,000-54,990$ \\
aOMAD & 0.0 & Single-loop & $264 \times 126 \times 60$ & $210,000-210,990$ \\
a7MAD & 0.7 & Single-loop & $264 \times 126 \times 60$ & $91,000-91,990$ \\
a9MAD & 0.9 & Single-loop & $264 \times 126 \times 60$ & $47,000-47,990$ \\
\hline
\end{tabular}

GRay achieves an order of magnitude speed up and allows us to compute $\mathcal{O}\left(10^{6}\right)$ images during $12 \mathrm{hr}$ of wall time using 32 nVidia Tesla K20X GPUs on the El Gato cluster at the University of Arizona.

In the next section, we describe the GRMHD simulations, the plasma methods, our implementation of the radiative processes, and the ray tracing algorithm that we are using in this paper. In Section 3, we study the dependence of the calculated spectra and images on the various model parameters. In Section 4, we compile the current spectral and imaging observations of Sgr A*, which we then use in Section 5 in order to constrain the range of model parameters. Finally, we discuss the implications of our results in Section 6.

\section{ACCRETION MODELS}

We carry out ray tracing calculations on six sets of threedimensional GRMHD simulation reported in Narayan et al. (2012) and Sądowski et al. (2013a), which consist of two different classes of accretion flows. The first class, called Standard And Normal Evolution (SANE), uses multi-loop initial magnetic fields, while the second class, called Magnetically Arrested Disk (MAD), uses single-loop initial fields. The accretion flows were evolved for exceptionally long times, e.g., $\sim 200,000 G M c^{-3}$, until the flows reached steady state up to $\sim 100 G M c^{-2}$. Three values of spin parameter, $a=0,0.7$, and 0.9 were used in each class. We summarize the setup of these simulations in Table 1.

\subsection{Characteristics of the MHD Simulations}

The differences in the initial magnetic fields and black-hole spins affect the dynamic and thermodynamic properties of the accretion flows. Because the plasma- $\beta$ and the gas temperature are important quantities in the emission models and affect the observables, we first calculate, for each simulation, their temporal and azimuthal averages, which we denote by

$$
\langle\langle\beta\rangle\rangle \equiv\left\langle\left\langle P_{\mathrm{gas}} / P_{\mathrm{mag}}\right\rangle\right\rangle
$$

and

$$
\langle\langle T\rangle\rangle \equiv\langle\langle u / \rho\rangle\rangle,
$$

and plot them in Figure 1. In the above definitions, $P_{\text {gas }}$ and $P_{\text {mag }}$ denote gas and magnetic pressure, while $u$ and $\rho$ denote the internal energy and density.

All simulations show a funnel region with low plasma- $\beta$ and high temperature, with only exception the zero spin SANE simulation aOSANE. This makes aOSANE a special configuration in our subsequent studies. The transition from the funnel region to the disk occurs over a rather narrow sheath, across which the 


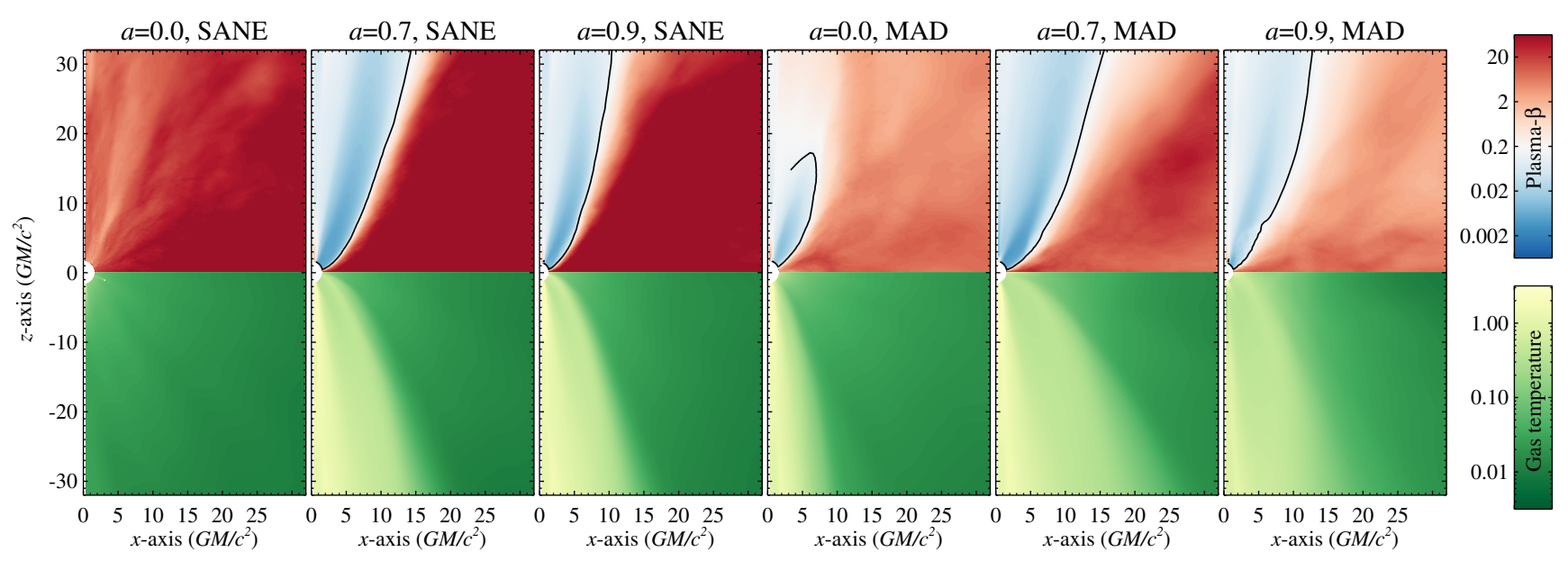

Figure 1. Top: temporal and azimuthal averages of the plasma- $\beta$ for the suite of GRMHD simulations listed in Table 1. The color scales for all the simulations are the same and shown in the right-most color bar. Blue represents $\langle\langle\beta\rangle\langle 0.2$ and shows the strongly magnetized regions. Red represents $\langle\langle\beta\rangle\rangle>0.2$ and shows the gas dominated regions. The black solid lines mark the $\beta=0.2$ contours. The saturated red regions around the equator of all the SANE simulation indicate that they are gas dominated, while the MAD simulations have stronger magnetic fields. Bottom: temporal and azimuthal averages of the comoving dimensionless gas temperature $\langle\langle T\rangle$. The color scales for all the panels are the same and shown in the right-most color bar. Green and yellow are low and high temperatures, respectively. Note that all simulations show a low plasma- $\beta$ /high temperature funnel region except for the non-spinning SANE simulation a0SANE. The simulations are described in detail in Narayan et al. (2012) and Sądowski et al. (2013a).

plasma- $\beta$ changes rapidly (note the logarithmic scale for the colors). This is consistent with the findings of Mościbrodzka $\&$ Falcke (2013) and allow us to separate the funnel from the disk by setting a threshold on the plasma- $\beta$, which here we fix to $\beta_{\text {threshold }}=0.2$ (see Section 5). The saturated red region around the equators of all the SANE simulations indicates that the accretion disks are strongly gas dominated. The MAD simulations, on the other hand, tend to have stronger magnetic fields even in the disk regions. For more details of the simulations, we refer to the original papers by Narayan et al. (2012) and Sachowski et al. (2013a).

We note that the coordinate singularities along the poles can cause numerical difficulties and can lead to a few low-density hot cells. Although these artificial hot cells do not affect the flow dynamics, they may change the predicted spectra by over producing $\mathrm{X}$-rays and $\gamma$-rays. In addition, the simulations use a special coordinate transformation developed by Tchekhovskoy et al. (2011) to "cylindrificate" the grid near the poles. While this technique significantly speeds up the GRMHD simulations by allowing a larger time step, it expands the problematic hot cells and enlarges the error. To overcome these numerical artifacts, we excise the cells around the poles by setting their emissivity to zero when they are unphysically hot compared to their neighbors.

The six numerical models described above are scale free in length and density (their time, velocity, and energy scales can be obtained by scaling length and density with different powers of the speed of light $c$ ). In order to compute images for Sgr A*, we fix the length scale by setting the mass of the central black hole to that of Sgr A*, $M=4.3 \times 10^{6} M_{\odot}$ (Gillessen et al. 2009). We introduce the density scale $n_{\mathrm{e}}$ as a free parameter to convert the gas density $\rho$ into the electron number density, which at the same time determines the physical accretion rate. We also define the observer's inclination angle $i$ with respect to the spin axis of the black hole and treat it as a free parameter.

\subsection{Thermodynamics of the Accretion Flow}

To calculate spectra, light curves, and images of the accretion flow around Sgr A*, we need to specify the electron temperature in the disk and in the funnel regions, which depend on the details of the heating due to the dissipation of turbulence, the radiative cooling, as well as the energy exchange between the protons and the electrons in the flow. In low density regions, where the collisional timescale is long compared to the dynamical timescale, the electron temperature $T_{\mathrm{e}}$ is expected to be lower than the ion temperature $T_{\mathrm{i}}$, leading to a two-temperature plasma (Shapiro et al. 1976; Narayan et al. 1995, 1998; see also the recent review by Yuan \& Narayan 2014 and references therein). In addition, in the funnel region, where the heating rate due to the dissipation of the MHD turbulence is expected to be low, thermal conduction can be efficient in bringing the electrons to a constant temperature (Mościbrodzka \& Falcke 2013; Mościbrodzka et al. 2014). We treat both of these possibilities in the calculation of the radiation from the accretion flow and use the following parameterizations to capture the resulting electron temperatures.

Constant electron-ion temperature ratio model.In the first model for the plasma, we assume that the electron-to-ion temperature ratio is a fixed function of the plasma- $\beta$. This is motivated by the fact that the temperature of the electrons can differ significantly from that of the protons as the gas density gets lower and the magnetic field strength, which influences the cooling time for the electrons via synchrotron emission, gets higher. We consider a simple step-function model where the electron temperature takes the following form:

$$
T_{\mathrm{e}} / T_{\mathrm{i}}= \begin{cases}\theta_{\mathrm{disk}} & \text { if } \beta>\beta_{\text {threshold }}, \\ \theta_{\text {funnel }} & \text { otherwise. }\end{cases}
$$

In this equation, the symbols $\theta_{\text {disk }}$ and $\theta_{\text {funnel }}$ denote the electron temperatures in the disk and in the funnel, in units of the ion temperature. Both quantities are expected to be less than unity under the assumptions that (1) dissipation of turbulence mainly heats up the ions, (2) the electrons cool faster than the ions, and (3) the electron cooling rate is faster than the energy exchange rate between electrons and ions (see discussion in Narayan \& Yi 1994, 1995; Quataert \& Gruzinov 1999). We specify the electron-to-ion temperature ratio in the disk, $\theta_{\text {disk}}$, and the electron-to-ion temperature ratio in the funnel, $\theta_{\text {funnel }}$, and constrain these parameters in the next section using observations of Sgr $\mathrm{A}^{*}$. Note that this model, which we will refer to as the "constant ratio model" in the remainder of the 
paper, reduces to the standard two-temperature plasma model used in earlier studies (e.g., Dexter et al. 2009; Mościbrodzka et al. 2009), if we fix $\theta_{\text {disk }}=\theta_{\text {funnel }}$.

Constant funnel electron temperature model.The second model accounts for the possible effects of electron conduction in the funnel region. We follow the parameterization in Mościbrodzka \& Falcke (2013) and assume that the electron temperature in the funnel is constant. We retain the constant temperature ratio parameterization for the disk. Because the plasma- $\beta$ is a good indicator of the separation between the highly magnetized, low density funnel region and the higher density disk, we use a threshold value of this parameter to specify the change in the electron temperature, such that

$$
T_{\mathrm{e}}= \begin{cases}T_{\mathrm{i}} \theta_{\mathrm{disk}} & \text { if } \beta>\beta_{\mathrm{threshold}}, \\ T_{\mathrm{e}, \text { funnel }} & \text { otherwise. }\end{cases}
$$

Note that $T_{\mathrm{e} \text {,funnel }}$ may acquire values larger than unity for ultrarelativistic electrons according to Equation (2). We vary the values of the threshold plasma- $\beta, \beta_{\text {threshold }}$, the electronto-proton temperature ratio in the disk, $\theta_{\text {disk }}$, and the electron temperature in the funnel, $T_{\mathrm{e} \text {,funnel }}$, to investigate the effects of these three parameters on the observables and when fitting the observed spectrum and images of Sgr A*. We refer to this as the "constant temperature model" in the rest of the paper.

\subsection{Radiative Processes and Transfer}

We calculate the radiation emitted from the accretion flow by solving the radiative transfer equation along null-geodesics through the domain of the GRMHD simulations, using the GRay code. GRay integrates the radiative equation backward from the image plane to the source. This approach has at least two advantages. First, it allows us to solve only for light rays that are normal to the image plane, drastically reducing the number of photon trajectories that need to be integrated. Second, integrating the radiative transfer equation backward allows us to stop the integration when the optical depth is sufficiently large (we use a cutoff at $\ln 1000 \approx 6.9$ ) or when the outgoing ray is sufficiently far away $\left(\sim 1000 G M c^{-2}\right)$ from the black hole. ${ }^{4}$ This significantly speeds up the image calculation for optically thick media. Both of these advantages make ray tracing algorithms much faster than Monte Carlo techniques for our application.

To ensure numerical stability, we follow Younsi et al. (2012) to express the radiative transfer equation in two coupled differential equations:

$$
\begin{aligned}
& \frac{d \tau}{d \lambda}=\gamma^{-1} \alpha_{0, v} \\
& \frac{d \mathcal{I}}{d \lambda}=\gamma^{-1}\left(\frac{j_{0, v}}{\nu^{3}}\right) e^{-\tau}
\end{aligned}
$$

where $\lambda$ is the affine parameter, $\gamma^{-1} \equiv \nu_{0} / \nu$ is the relative energy shift, $\tau$ and $\mathcal{I}$ are the optical depth and Lorentz invariant intensity at frequency $v$, and $\alpha_{0, v}$ and $j_{0, v}$ are comoving absorption and emission coefficients.

In hot, magnetized accretion flows, synchrotron and bremsstrahlung radiation are the two major radiative processes that contribute to the emission and absorption (see Narayan

\footnotetext{
4 The actual criterion is available in the GRay source code, https://github.com/chanchikwan/gray. All results presented in this paper should be reproducible (within round-off error) by using commit 0c99a24c and CUDA 6.0 .1 .
}

et al. 1998). In this paper, we assume thermal synchrotron and bremsstrahlung emission and do not treat the possible contribution from non-thermal electrons, which can contribute to both radio and X-ray fluxes (Mahadevan 1998; Özel et al. 2000) and can help explain the variability observed in the X-rays (Chan et al. 2009). For synchrotron emissivities, we use the approximate expression derived by Leung et al. (2011). For thermal bremsstrahlung emission, we use the expression derived in Rybicki \& Lightman (1979), with a Gaunt factor taken from Novikov \& Thorne (1973). ${ }^{5}$ Although Compton scattering is generally important in stellar-mass black holes, its contribution to the Sgr $A^{*}$ spectrum is mainly in the optical (see, e.g., Narayan et al. 1998), for which we do not have data to impose any constraints on (see Section 4). Moreover, Mościbrodzka et al. (2009, see their Figure 4) showed that the contribution of Compton scattering to the X-ray flux predicted in GRMHD simulations is typically much smaller than the observed flux, unless the black hole is rapidly spinning and observed from a nearly edge-on orientation. For these two reasons, we neglect here the effects of Compton scattering on the spectrum.

\section{SPECTRAL AND IMAGE PROPERTIES}

Using GRay for radiation transport and the plasma models described in the previous section, we calculate the broadband spectra emerging from the accretion flow in each GRMHD simulation. We compute a radiation spectrum from a series of snapshots taken from the GRMHD simulations. In order to take into account the emission from near the event horizon as well as at the outer parts of the computational domain, we compute the images using three different fields of view: the innermost images cover a $32 r_{\mathrm{S}} \times 32 r_{\mathrm{S}}$ plane, the middle images cover a $128 r_{\mathrm{S}} \times 128 r_{\mathrm{S}}$ plane, and the outermost images cover a $512 r_{\mathrm{S}} \times 512 r_{\mathrm{S}}$ plane, where $r_{\mathrm{S}} \equiv 2 G M c^{-2}$ is the Schwarzschild radius. The outermost image size is chosen so that the $\mathrm{X}$-ray fluxes computed within the simulation volume converge. We resolve each domain using $512 \times 512$ pixels and obtain the total flux by appropriately combining and summing the contributions from the three images.

In order to explore the effect of the parameters describing the thermodynamics of the plasma, as well as of the inclination of the observer with respect to the spin axis of the accretion flow, on the spectra and images of the flows, we first calculated a large suite of simulations for a range of values of the parameters. Each of the two plasma models described in the previous section requires five parameters to be fully specified. For the constant ratio model, these are: the normalization of the electron density $n_{\mathrm{e}}$, the inclination of the observer $i$, the threshold plasma- $\beta$, $\beta_{\text {threshold }}$, that separates the funnel from the disk, the electron-toion temperature ratio in the funnel, $\theta_{\text {funnel }}$, and the electron-to-in temperature ratio in the disk, $\theta_{\text {disk }}$. For the constant temperature model, these are: the normalization of the electron density $n_{\mathrm{e}}$, the inclination of the observer $i$, the threshold plasma- $\beta, \beta_{\text {threshold }}$, the funnel electron temperature $T_{\mathrm{e}, \text { funnel }}$, and the electron-to-ion temperature ratio, $\theta_{\text {disk }}$ in the disk.

In Figure 2, we show the effect of independently varying each of these parameters on the simulated radio-to-X-ray spectrum for Sgr A*. Each column corresponds to one of the GRMHD simulations listed in Table 1, while each row shows how the spectra change in response to varying one of the five model

\footnotetext{
5 Although Rybicki \& Lightman (1979) cited Novikov \& Thorne (1973) for their Gaunt factor, the actual formulae are different. See the GRay source code for our implementation of the Gaunt factor.
} 


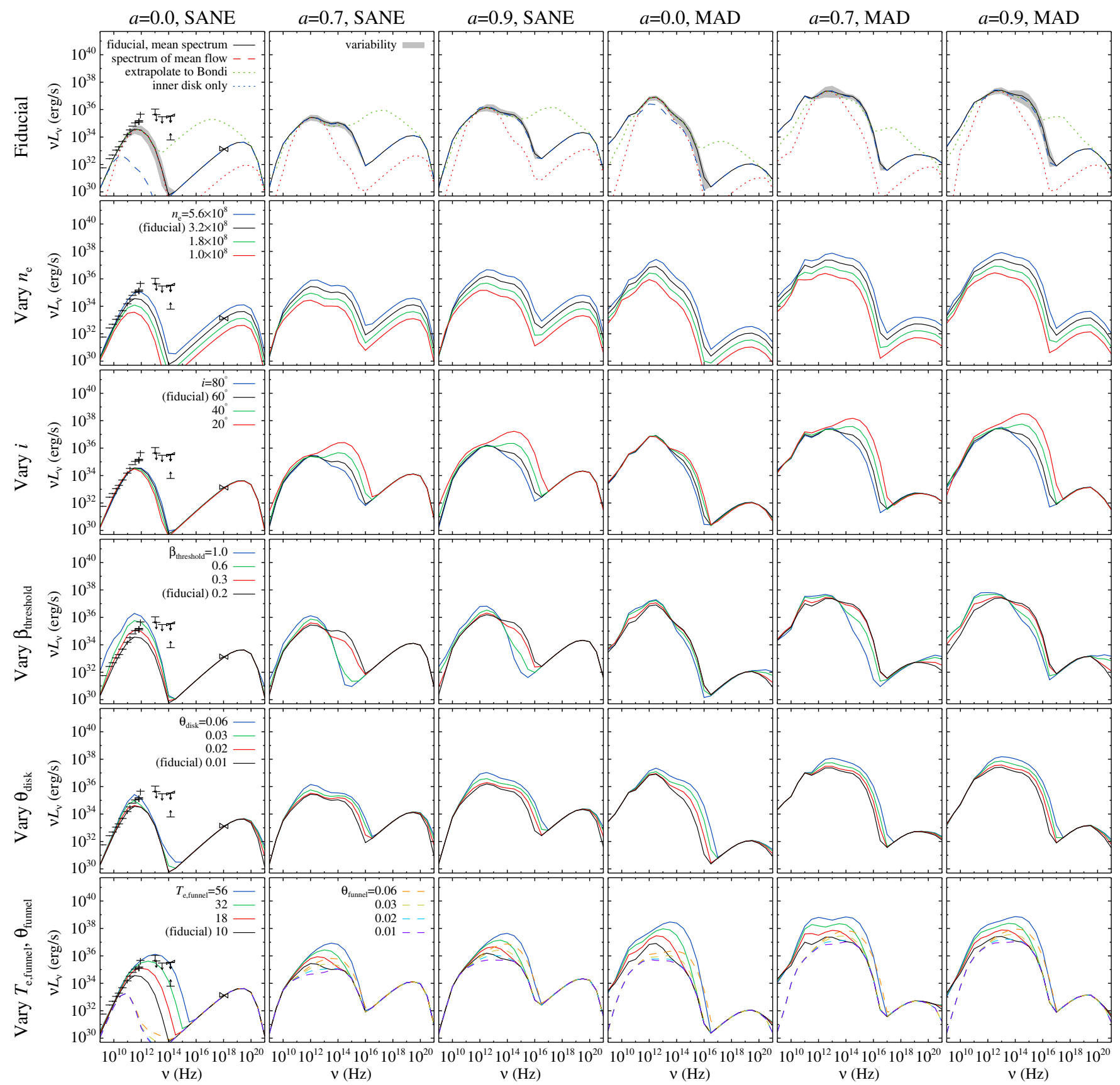

Figure 2. Broadband spectra computed for the suite of GRMHD simulations listed in Table 1. Each column corresponds to a different simulation, while each row shows the effect of varying one of the model parameters. The data points in the first column correspond to various observations of Sgr A*. The top "Fiducial" row uses parameters $i=60^{\circ}, n_{\mathrm{e}}=3.2 \times 10^{8}, \beta_{\text {threshold }}=0.2, \theta_{\text {disk }}=0.01$, and $T_{\mathrm{e} \text {,funnel }}=10$. In each panel, the shaded gray area marks the variability of spectra computed from 100 snapshots; the black solid curve marks their average; the red dotted curve marks the average spectrum of the inner accretion flow; the green dotted curve marks the average spectrum computed after extrapolating the properties of the flow out to the Bondi radius; and the blue dashed curve marks the spectrum of the mean flow. We vary the normalization of the electron density $n_{\mathrm{e}}$ in the second row, the observer's inclination $i$ in the third row, the threshold plasma- $\beta$ in the fourth row, and the electron-ion temperature ratio $\theta_{\text {disk }}$ in the disk in the fifth row. In the sixth row, we either vary the funnel electron temperature $T_{\mathrm{e}, \text { funnel }}$ (solid curves) or the funnel electron-ion temperature ratio $\theta_{\text {funnel }}$ (dashed curves).

parameters, while keeping the others fixed. The observational data points shown in the leftmost panels were collected by Broderick et al. (2011a).

As mentioned at the beginning of this section, the X-ray fluxes computed within the simulation volume converge at a radius of $\sim 512 r_{\mathrm{s}}$. However, this radius is much smaller than the Bondi radius of Sgr $\mathrm{A}^{*}, \sim 10^{5} r_{\mathrm{S}}$, at which the accretion flows is expected to still have significant contribution to the $\mathrm{X}$-ray emission (see, e.g., Quataert 2004). Our models do not include this large volume of X-ray emission because the central blackhole is fed by a torus that lies at a few hundred Schwarzschild radii. To consider the contribution of the large scale flow, we computed a set of spectra up to the Bondi radius by using the extrapolation scheme developed in Sądowski et al. (2013b). The results are shown as green dotted curves in the first row in Figure 2. The X-ray fluxes of the extrapolated flows are oneto-two orders of magnitude larger, as expected. Note that even though we show the results from this extrapolation here, we will 
opt to limit our domain to the inner accretion flow and consider the fraction of X-rays that originate from the inner flow when actually fitting the X-ray data to the models (see Section 4).

Fiducial model. The resulting broadband spectrum for each GRMHD simulation and a "fiducial" plasma model with a representative set of parameters $\left(n_{\mathrm{e}}=3.2 \times 10^{8}, i=60^{\circ}\right.$, $\beta_{\text {threshold }}=0.2, \theta_{\text {disk }}=0.01$, and $\left.T_{\mathrm{e}, \text { funnel }}=10\right)$ is depicted in the first row of Figure 2. Note that this model is not a specific fit to Sgr A* observations. We use the fiducial setup, before embarking on fitting the observations, in order to study several theoretical aspects of the spectra, such as the predicted variability across the spectrum and the different emission regions that give rise to the structures of the images at different wavelengths.

The strong turbulence in the accretion flow naturally causes the resulting spectra and images to be time dependent. This complicates the comparison of the simulations to the observations, as both need to be averaged properly in order to avoid comparing a particular realization of the turbulent flow in the simulations to a different realization in the observed flow. In order to assess the variability of the simulated spectra and images we perform our ray tracing calculation for 100 different snapshots in each of the simulations, as listed in Table 1. Each snapshot of the simulation was obtained at regular time intervals of $10 \mathrm{GMc} c^{-3}$. For the mass of Sgr A*, 100 snapshots correspond to $\approx 5.9 \mathrm{hr}$, which is similar to the time interval over which EHT observations will take place.

The black solid curves on the panels of the first row in Figure 2 show the mean spectra obtained by averaging the simulated spectra in the 100 snapshots of each simulation. The shaded gray area around them marks the maxima and minima of the spectra emerging from these 100 snapshots and is representative of the expected spectral variability. The red dotted curves on the same panels show the average spectra computed using only the image of size $32 r_{\mathrm{S}} \times 32 r_{\mathrm{S}}$, which we refer to as the emission from the inner accretion flow. As expected, when the emission originates from the inner accretion flow, the spectra acquire their largest degree of variability while the opposite is true when the emission originates in a much larger volume. Indeed, in the optically thick, low-frequency region of the spectrum, the emission is weakly variable because the radius of the photosphere at these frequencies is equal to tens to hundreds of Schwarzschild radii and the dynamical timescales there are very long. The variability is also weak at the optically thin, high-frequency region of the spectrum, where the emission is generated by thermal bremsstrahlung over a very large volume around the black hole. In the millimeter to IR range (i.e., $v \sim 10^{11}-10^{14} \mathrm{~Hz}$ for the aOSANE simulation), however, the emission is optically thin and originates very close to the event horizon. The characteristic timescales there are very short and both the spectra and images show significant variability.

To visualize this point in a different manner, we show in Figure 3 the averaged snapshot images of the a9MAD simulation, for the fiducial parameters of the plasma model. The different rows, from top to bottom, depict images at frequencies $v=$ $10^{11} \mathrm{~Hz}, 10^{13} \mathrm{~Hz}$, and $10^{18} \mathrm{~Hz}$. The different columns, from left to right, are for image sizes equal to $512 r_{\mathrm{S}} \times 512 r_{\mathrm{S}}$, $128 r_{\mathrm{S}} \times 128 r_{\mathrm{S}}$, and $32 r_{\mathrm{S}} \times 32 r_{\mathrm{S}}$. As discussed above, the emission at the lowest and highest frequencies shown originates primarily from large distances away from the black hole. On the other hand, the emission at infrared frequencies originates in a very small region, close to the horizon. The black-hole shadow is obscured at the optically thick radio frequencies but is visible in both infrared and X-ray, which are optically thin. For the $\mathrm{X}$-ray images, although the region around the black-hole shadow has a larger surface brightness than all other regions, most of the (integrated) flux actually originates from a few hundred Schwarzschild radii in our models.

In calculating the average spectra and images discussed above, we computed individual spectra and images for each snapshot of the simulations and then averaged together the resulting surface brightness and fluxes on the image plane of the observer. This procedure generates results that can be very different compared to calculating the average hydrodynamic and thermodynamic properties of each simulation and then computing a single image and spectrum for this mean flow. This is because of the fact that the plasma properties are substantially variable and the radiative transfer equation, which we solve along geodesics to calculate the image brightness, is a highly non-linear function of the plasma properties. In the top row of Figure 2, we show as blue dashed curves the spectra computed using the mean properties of the flows. The relative difference between the two averaging procedures is largest in the case of the non-spinning black holes.

In Figure 4, we compare the images computed by the two averaging approaches for a frequency of $10^{11} \mathrm{~Hz}$, using the aOSANE simulation. The left panel shows the image calculated using the mean properties of the flow and the right panel shows the average of the images in each snapshot. There is a striking difference between the two images. The image of the mean flow (left panel) is very dim and almost invisible in the plot. On the other hand, the average image of the snapshots is dominated by magnetic filaments, which are short lived (and hence do not contribute significantly to the average properties of the flow) but are very bright (and hence dominate the average emission).

Finally, comparing the spectra calculated for the different GRMHD simulations but for the same, fiducial plasma model, we find that the flux at the thermal peak increases monotonically as we move from the leftmost to the rightmost columns. In other words, there is more low-frequency emission in the MAD and in the high-spin simulations. This is a direct consequence of the fact that MAD and high-spin simulations are characterized by significant relativistic jets. In contrast, the MAD simulations generate less X-ray radiation than the SANE simulations because there is less flux from the accretion flows at large radii (i.e., $r \gtrsim 128 r_{\mathrm{S}}$ ). This is also a direct consequence of the fact that the density in the MAD simulations is more centrally concentrated than in the SANE simulations (see Narayan et al. 2012).

Parameter study. The remaining rows in Figure 2 show how the spectra are affected when we vary one of the parameters of the plasma model, i.e., $n_{\mathrm{e}}, i, \beta_{\text {threshold }}, T_{\mathrm{e} \text {,funnel }}, \theta_{\text {disk }}$, and $\theta_{\text {funnel }}$, while holding all other parameters fixed to the fiducial model.

We first vary the density scale in the range $n_{\mathrm{e}}=10^{8}$ to $n_{\mathrm{e}}=$ $5.6 \times 10^{8}$ and show the result for each GRMHD simulation in the second row of Figure 2. At frequencies $v \gtrsim 10^{12} \mathrm{~Hz}$, increasing the density scales causes an increase in the overall flux that is proportional to $n_{\mathrm{e}}^{2}$. This dependence is trivial to understand for the X-rays, which are generated by optically thin bremsstrahlung emission, since the emissivity of bremsstrahlung scales as the square of the electron density. In the infrared, near the peak of the thermal bump, the emission is primarily due to optically thin synchrotron processes, with an emissivity that scales as $j_{\text {synch }} \propto n_{\mathrm{e}} B^{2}$. However, the natural scaling of the GRMHD equations causes the dimensional magnetic field strength in the flow to be always proportional to the square root of the 


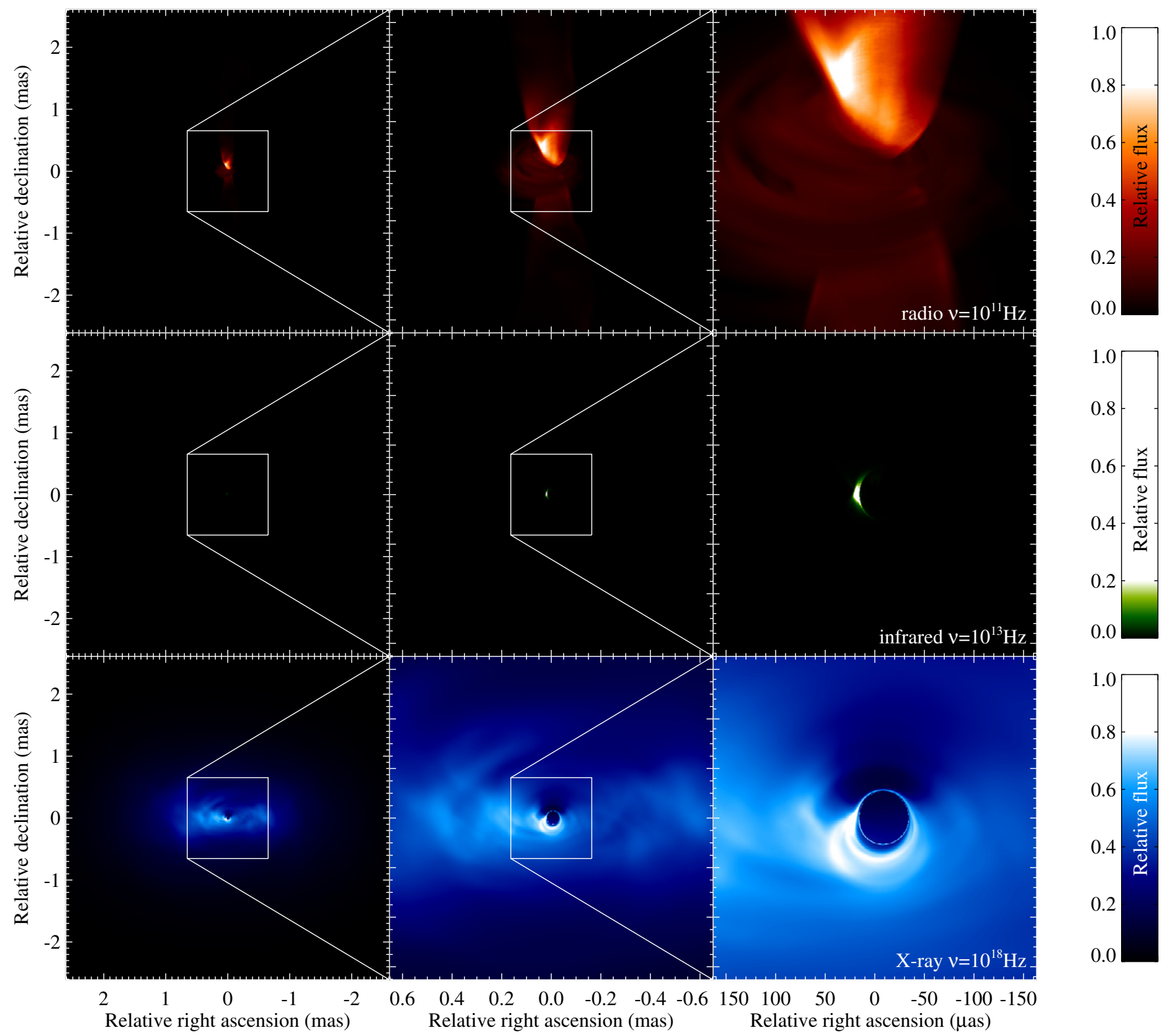

Figure 3. Simulated images of Sgr A* at three frequencies using the simulation a9MAD with the fiducial model parameters. The color scale for each row is shown on the right, with unity being the maximum flux (the same color scales are used in the subsequent image plots). The different columns, from left to right, depict images computed on different zoom scales of $512 r_{\mathrm{S}} \times 512 r_{\mathrm{S}}, 128 r_{\mathrm{S}} \times 128 r_{\mathrm{S}}$, and $32 r_{\mathrm{S}} \times 32 r_{\mathrm{S}}$. In order to preserve individual visible features in the accretion flows, we average only the 10 last snapshots, i.e., between $t=47,900$ and 47,990. The low frequency radio and the X-ray emission originate from large volumes, whereas the infrared emission primarily arises from the inner accretion flow.

electron density. In other words, $B \propto \sqrt{n_{\mathrm{e}}}$, and the optically thin synchrotron emissivity also scales as $j_{\text {synch }} \propto n_{\mathrm{e}}^{2}$.

At lower frequencies, i.e., when $v \lesssim 10^{12} \mathrm{~Hz}$, the emerging flux has a weaker dependence on the density scale. In this part of the spectrum, the inner accretion flow becomes optically thick to synchrotron self absorption. Increasing the density scale causes the cooler outer disk to partially block the hotter inner disk and, therefore, to flatten the dependence of the flux on the electron density. To demonstrate this point, we show in Figure 5 the images at a frequency of $v=10^{11} \mathrm{~Hz}$ for the a7SANE simulation and for the four different values of the electron density scale. As we increase the density scale, the inner accretion disk and the funnel become brighter as expected. An increasing fraction of that bright emission, however, is obscured by the colder outer disk, reducing the strong dependence of the flux on the scale of the electron density.

In the third row of Figure 2, we show the effect of varying the inclination of the observer in the range $i=20^{\circ}$ to $i=80^{\circ}$. At low frequencies, i.e., when $v \lesssim 10^{12} \mathrm{~Hz}$, the flux has a very weak dependence on the inclination. The dependence becomes stronger, however, at somewhat higher frequencies. In order to understand this behavior, we show in Figure 6 the corresponding images of the accretion flow in the a9SANE simulation. At low frequencies (top row), as the observer's inclination increases, a larger fraction of the hot inner flow is obscured by the colder outer disk. This effect cancels out the fact that both the upper and lower funnel regions become visible, and produces an overall weak dependence of the inclination. At intermediate frequencies 


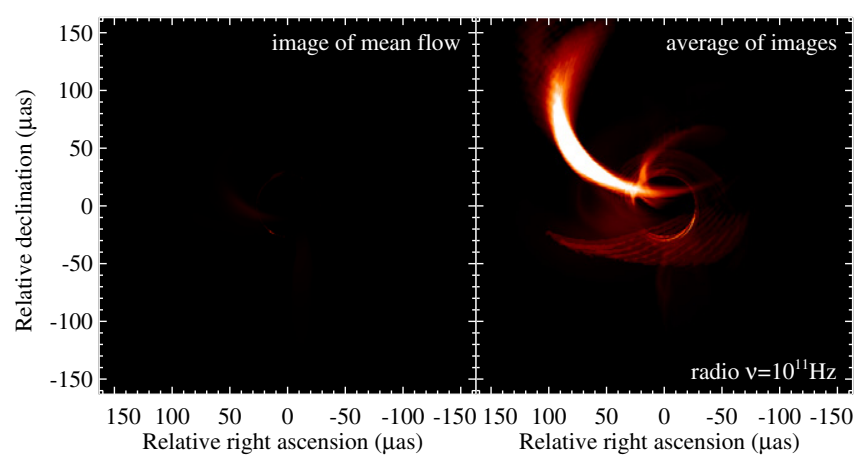

Figure 4. Comparison of the predicted image at $v=10^{11} \mathrm{~Hz}$ using the average properties of the simulation (left) to the average of individual images computed for each snapshot of the simulation (right). For this figure, we used the aOSANE simulation with the parameters of the fiducial model. The striking difference between the images is due to the presence of short-lived, magnetically dominated filaments in the inner accretion flow, which contribute significantly to the emission in each snapshot but are washed out when computing the mean flow.

(bottom row), the optically thin emission originates very close to the black-hole shadow; increasing the inclination causes the Doppler effect to boost more radiation toward the observer and, therefore, increases the resulting flux. In X-rays (not shown in the figure), which originate in a large, quasi-spherical volume, there is very little dependence of the resulting spectrum on the inclination.

In the fourth row of Figure 2, we show the effect of varying the threshold plasma- $\beta$ that distinguishes the disk from the funnel, in the range $\beta_{\text {threshold }}=0.2$ to $\beta_{\text {threshold }}=1.0$. For the parameters of the fiducial model, the funnel is brighter than the disk in the radio to infrared frequencies. Therefore, increasing $\beta_{\text {threshold }}$, which creates a larger funnel, increases the radio and infrared flux for all simulations. The situation is reversed, however, at higher frequencies, where the emission is dominated by the disk as shown in the lower row of Figure 6. Note also that, primarily in the MAD simulations, the $\gamma$-ray flux is also affected by the threshold value of the plasma- $\beta$. This arises from the few hot zones along the pole near the event horizon, as we described in Section 2.2, and we have simply left it here to demonstrate this numerical artifact.

In the fifth row of Figure 2, we show the effect of varying the electron-to-ion temperature ratio in the disk, in the range $\theta_{\text {disk }}=0.01$ to $\theta_{\text {disk }}=0.06$. As expected, because the synchrotron and bremsstrahlung emissivities depend on the electron temperature, the flux in the optically thin part of the radio-to-infrared spectrum increases with $\theta_{\text {disk }}$.

Finally, in the sixth row of Figure 2, we show the effect of varying the thermodynamic properties of the electrons in the funnel. In the case of the constant temperature model, we vary the electron temperature in the funnel in the range $T_{\mathrm{e} \text {,funnel }}=10$ to $T_{\mathrm{e} \text {,funnel }}=56$; in the case of the constant ratio model, we vary the electron-to-ion temperature ratio in the funnel in the range $\theta_{\text {funnel }}=0.01$ to $\theta_{\text {funnel }}=0.06$. As discussed above, for the fiducial values of the parameters, the emission from the funnel dominates the radio-to-infrared part of the emission. As a result, changing the electron temperature in the funnel has a very large effect on that part of the spectrum. On the other hand, the X-ray emission comes primarily from the outer disk and, therefore, is not affected by the thermodynamic properties of the electrons in the funnel.

\section{CURRENT SPECTRAL AND IMAGING OBSERVATIONS OF Sgr A*}

In Section 3, we performed an extensive parameter study aiming to understand the dependence of our results on the model assumptions related to the MHD and thermodynamic properties of the accretion flow. Our next goal is to identify the set of models and parameters that are consistent with the current spectral and imaging observations of Sgr $\mathrm{A}^{*}$. The data points in the leftmost panels of Figure 2 correspond to non-simultaneous observations of $\mathrm{Sgr} \mathrm{A}^{*}$ at a broad range of frequencies and with different instruments. Several different, partially overlapping data sets exist in the literature. The particular data points we are using here were prepared by Broderick et al. (2011a), while a comparable collection of data is available in the more recent review by Falcke \& Markoff (2013).

When comparing the results of our simulations to spectral observations, we make the following choices.

1. In the radio, we do not consider the data points at frequencies below $10^{11} \mathrm{~Hz}$, because even a small fraction of non-thermal electrons in the accretion flow (which we do not include here) can affect significantly the flux at these low frequencies (see Mahadevan 1998; Özel et al. 2000; Chan et al. 2009).

2. In the infrared, i.e., at frequencies $10^{13} \mathrm{~Hz}<v<10^{15} \mathrm{~Hz}$, we do not consider the individual data points but rather

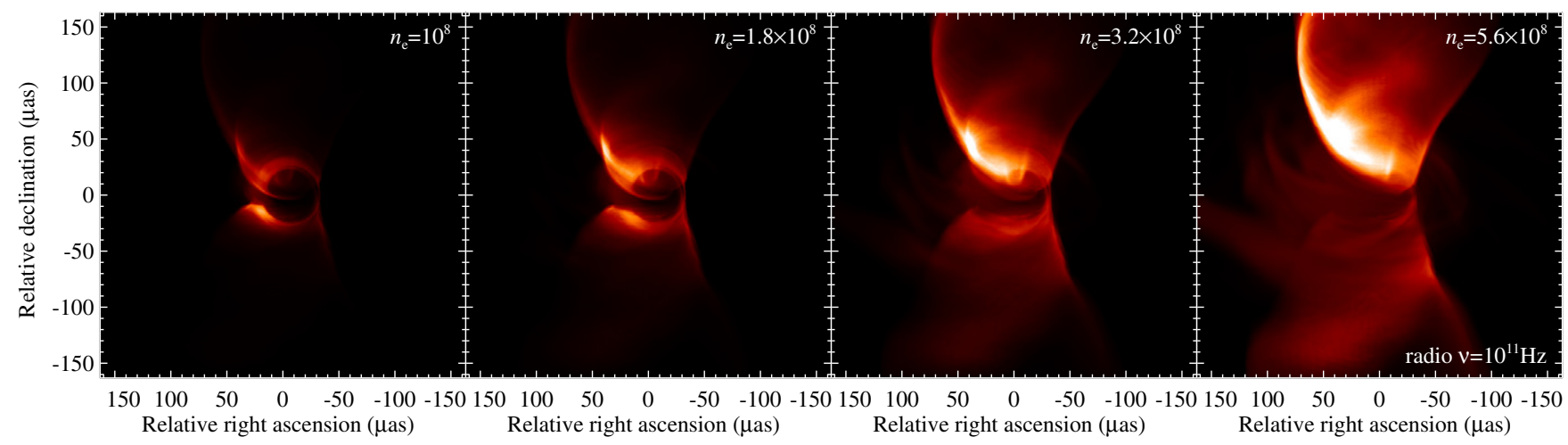

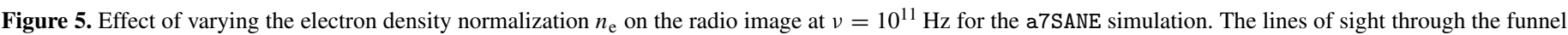

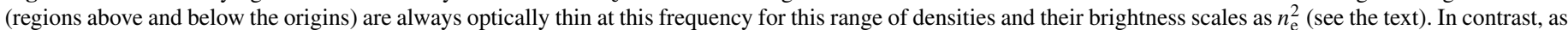

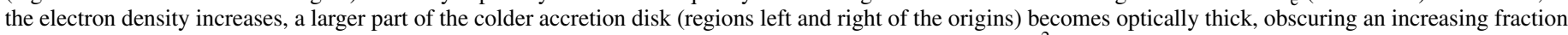
of the funnel. The net effect is a dependence of the overall flux on electron density scale that is weaker than $n_{\mathrm{e}}^{2}$ at this frequency. 


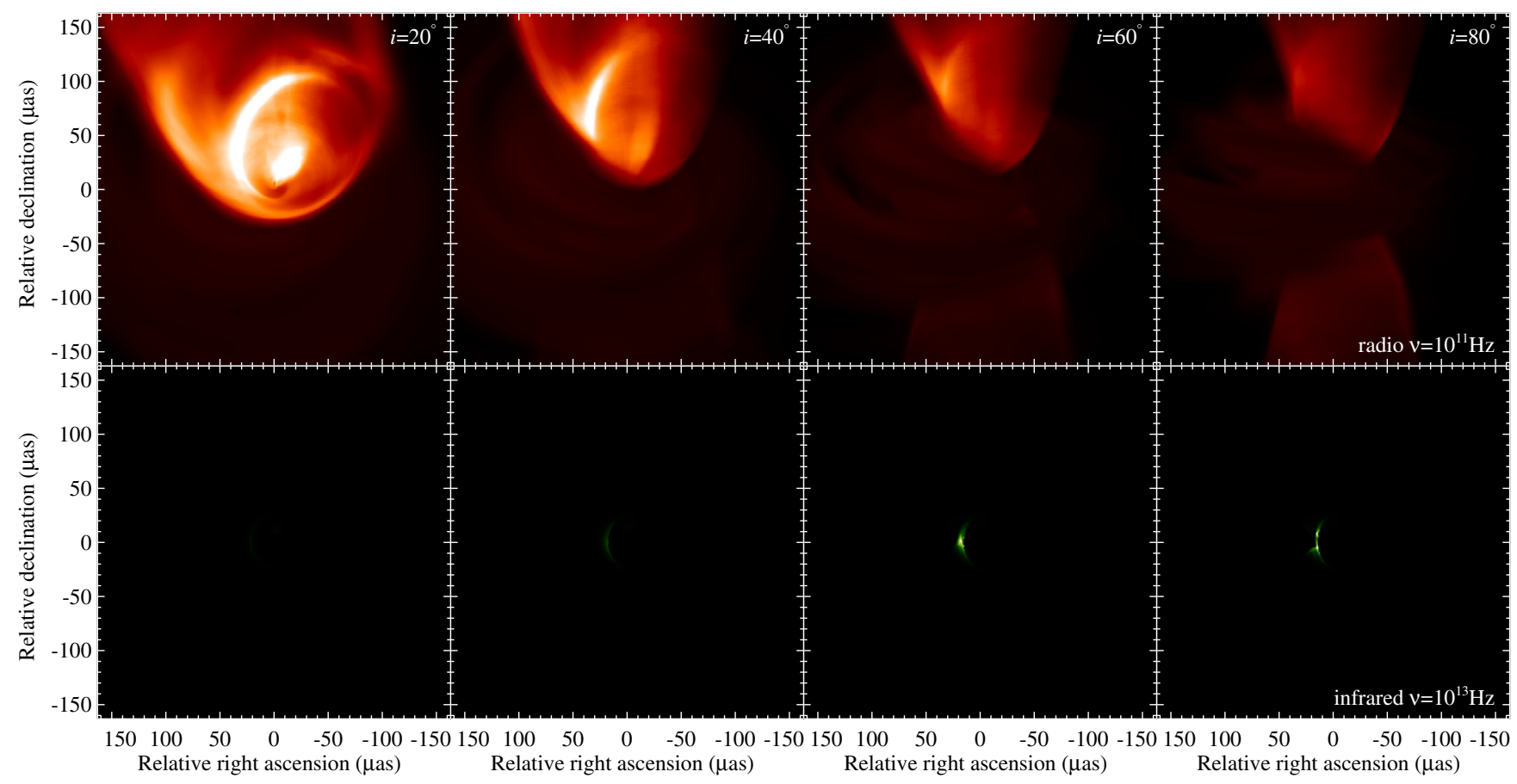

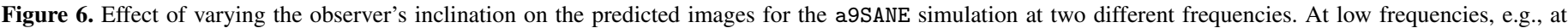

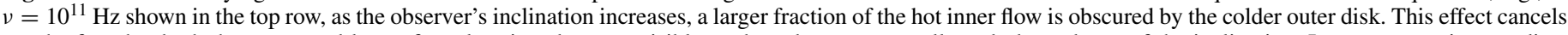

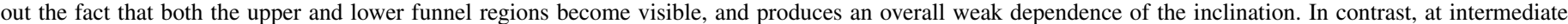

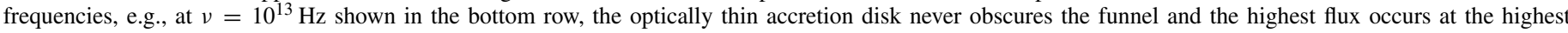

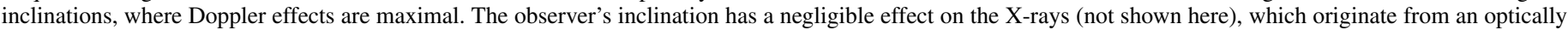
thin, quasi-spherical large volume.

require the simulated spectra to fall within the lowest and highest observed fluxes at $v=1.38 \times 10^{14} \mathrm{~Hz}$. This is justified by the fact that both the observational data (Genzel et al. 2003; Ghez et al. 2004; Dodds-Eden et al. 2011) and our simulations shown in Figure 2 indicate that the infrared emission is highly variable and specific observed or simulated fluxes will depend entirely on the specific realization of the turbulent flow.

3. In the X-rays, we investigated the option of extrapolating the accretion flow out to the Bondi radius following the prescription of Sadowski et al. (2013b, see also discussion of Figure 2) and computing the X-ray emission using this entire volume. We found, however, that our results depended very strongly on the assumed power-law indices of the density and temperature profiles as well as on the choice of the point at which the extrapolation matched the numerical solutions. Instead of following this approach, we opt to use the result of Shcherbakov \& Baganoff (2010, see also the discussion in Neilsen et al. 2013) that $10 \%$ of the quiescent $\mathrm{X}$-ray flux from $\mathrm{Sgr} \mathrm{A}^{*}$ originates in a point source and attribute $10 \%$ of the observed flux to the emission from our simulated volume.

The size of the image of $\operatorname{Sgr} \mathrm{A}^{*}$ has been measured over many wavelengths, from the radio to the millimeter (see Falcke \& Markoff 2013 for a recent review). At most wavelengths, the size measurement is dominated by the blurring of the image caused by interstellar scattering. There is strong evidence that, at wavelengths below $1 \mathrm{~cm}$, the intrinsic size of Sgr $\mathrm{A}^{*}$ can be discerned (see, e.g., Bower et al. 2014). However, because of the $\lambda^{2}$ dependence of the size of the scattering ellipse, the most accurate measurements occur at the smallest wavelengths. Early EHT observations of Sgr A* at $1.3 \mathrm{~mm}$ measured its size at $43_{-8}^{+14} \mu$ as. Correcting for the blurring using the scattering law of Bower et al. (2006) resulted in an inferred intrinsic size of the source equal to $37_{-10}^{+16} \mu$ as (Doeleman et al. 2008).

The inferred image size for $\mathrm{Sgr} \mathrm{A}^{*}$ was based on fitting sparse visibility data in the interferometric $u-v$ plane with a Gaussian model (alternate models have also been considered; see Doeleman et al. 2008). The images from our simulations, however, have significant asymmetry, either because they are dominated by emission in the funnel or because of Doppler effects in the disk. In principle, in order to compare our simulations to the observed image size, we will need to calculate the predicted scattering broadened visibilities in the $u-v$ plane and compare them directly to the data (as is done, e.g., in Broderick et al. 2011a and in Dexter et al. 2009). However, this introduces additional free parameters in the model, such as the orientation of the black-hole spin vector on the plane of the sky, and the current coverage of the $u-v$ plane is too sparse to allow us to constrain the model parameters significantly better than the simple estimate of the size (see, e.g., the large areas within the confidence contours in Broderick et al. 2011a and in Dexter et al. 2009). For this reason, we follow a more approximate procedure in comparing our simulations to the current estimates of the image size at $1.3 \mathrm{~mm}$.

We take into account the effects of interstellar scattering using the elliptical scattering kernel of Bower et al. (2006) that has a major axis

$$
\mathrm{FWHM}_{\text {major }}=1.309(\lambda / 1 \mathrm{~cm})^{2} \text { mas, }
$$

and a minor axis

$$
\mathrm{FWHM}_{\text {minor }}=0.64(\lambda / 1 \mathrm{~cm})^{2} \text { mas, }
$$

with a position angle of the major axis at PA $=78^{\circ}$ East of North. Because we do not know a priori the position angle of 


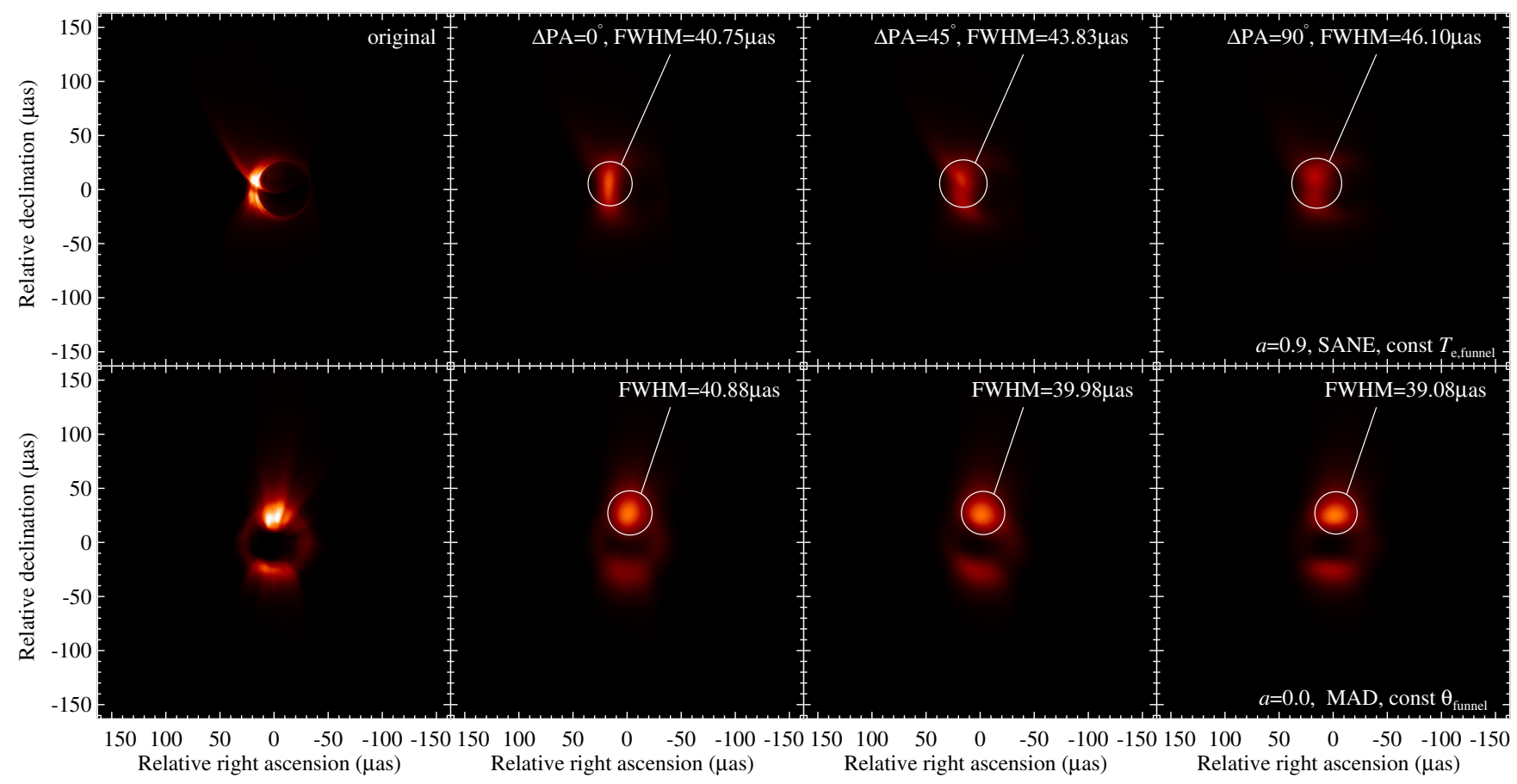

Figure 7. Calculated images at $1.3 \mathrm{~mm}$ for (top) the aOSANE simulation using the constant ratio model and (bottom) the a9SANE simulation using the constant temperature model. All of the parameters of the plasma model correspond to the best-fit configurations in each case (and not to the fiducial model, as in the previous figures). The leftmost panels are the results of the direct ray tracing calculations. The remaining columns show the effect of broadening caused by interstellar scattering. Because the relative position angle, $\Delta \mathrm{PA}$, between the spin axis of the black hole and the major axis of the scattering kernel is not known, the three columns show the resulting images for three different relative position angles as examples.

the black-hole spin axis, we consider the entire range of relative position angles between the major axis of the scattering kernel and the spin axis, $\triangle \mathrm{PA}$. For each configuration, we convolve the ray traced image from the simulation with the scattering kernel and fit the resulting blurred image with a single Gaussian profile.

In Figure 7, we show how the relative angle $\triangle \mathrm{PA}$ between the black-hole spin and the major axis of the scattering kernel affects the blurred image sizes. The top row shows $1.3 \mathrm{~mm}$ images for the aOSANE simulation, using the constant ratio model; the bottom row shows $1.3 \mathrm{~mm}$ images for the a9SANE simulation using the constant temperature model. Note that, in anticipation of the results presented in the following section, the plasma parameters for these two sets of images correspond to the best-fit values for Sgr A*, which we are going to obtain in the following section.

In each row, the first column shows the results of the direct ray tracing simulations. The remaining columns show the same image after taking into account the effects of interstellar scattering, for three values of the relative position angles. The dependence of the inferred image size on this parameter is weak. Moreover, in some cases, because of the asymmetries in the images, the blurred image size is not a monotonic function of the relative position angle. For this reason, for each simulation and for each set of model parameters, we scan the entire range of relative orientation angles and consider the range of predicted sizes as an uncertainty in the model. We then compare the predicted values to the observed image size of $43_{-8}^{+14} \mu$ as.

\section{CONSTRAINTS ON SGR A*}

For each of the GRMHD simulations, which corresponds to different black-hole spins and magnetic field configurations, and for either the constant ratio or the constant temperature models, we use observations described in the last section to constrain the five additional model parameters. In particular, we use, (1) the quiescent flux in the X-rays, (2) the observed flux and spectral shape in the frequency range $10^{11} \mathrm{~Hz} \leqslant v \leqslant 10^{12} \mathrm{~Hz}$, (3) the range of observed fluxes at $v \approx 1.3 \times 10^{14} \mathrm{~Hz}$, and (4) the image size at $1.3 \mathrm{~mm}$. This is a large parameters space, which is difficult to explore computationally and may lead to large degeneracies between model parameters.

Following Mościbrodzka \& Falcke (2013), we fix the threshold plasma- $\beta$ value that separates the disk from the funnel to $\beta_{\text {threshold }}=0.2$. Changing the value of this parameter would simply lead to correlated changes in the electron-to-ion temperature ratio in the disk, without affecting significantly the overall results. We take advantage of the fact that the spectra are insensitive to the inclination angle at low frequencies (see the third row of Figure 2) and set $i=60^{\circ}$ (this value was justified by Psaltis et al. 2014, and will be checked later for consistency). Finally, as we discussed in Section 3, several aspects of the simulated spectra and images depend very weakly on some of the model parameters. This allows us to follow the procedure described below, which leads us to use different aspects of the observations to constrain successive subsets of the remaining model parameters.

Fixing the electron density scale using the X-ray flux. In Figure 2, we showed that, among the five model parameters, the predicted $\mathrm{X}$-ray flux at $v \approx 10^{18} \mathrm{~Hz}$ for each simulation is only sensitive to the density normalization $n_{\mathrm{e}}$ when we fix the inclination. This property lets us find a correlation between $n_{\mathrm{e}}$ and $\theta_{\text {disk }}$ (with $\theta_{\text {funnel }}=T_{\text {e,funnel }}=0$ ) such that the simulated $\mathrm{X}$-ray flux agrees with the observed quiescent flux. The result is shown as a set of solid curves in the second row of Figure 8. Note that, because the accretion rates, in code units, are 


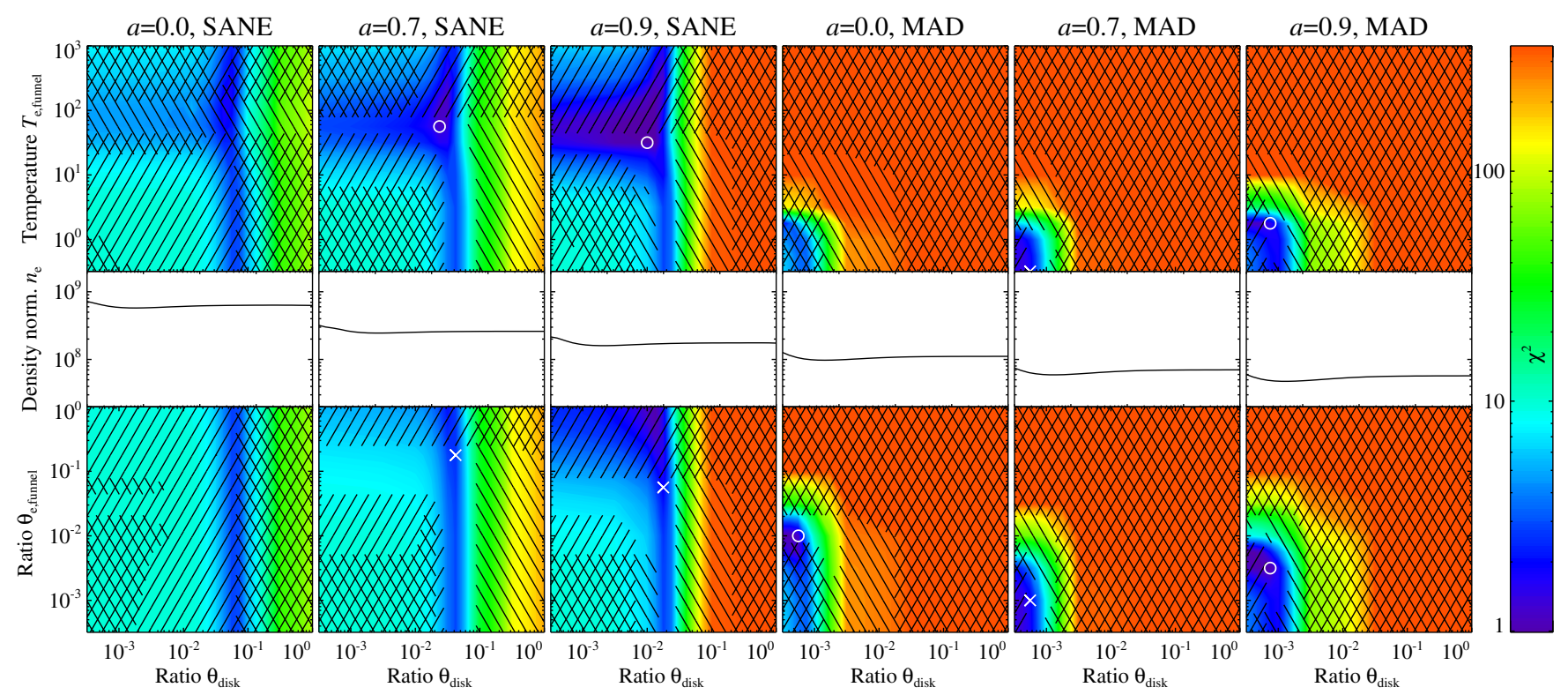

Figure 8. Constraints on model parameters obtained by comparing the results of simulations to the observed spectra and image sizes of Sgr $\mathrm{A}^{*}$, as described in the text and shown in Figures 9. Each column above corresponds to a different GRMHD simulation, while the top and bottom panels correspond to the two different descriptions of the plasma thermodynamics in the funnel. The solid curves in the middle row depict the electron density normalization for which the predicted $\mathrm{X}$-ray fluxes agree with $10 \%$ of the observed quiescent flux. The colored contour plots show values of $\chi^{2}$ (see the color bar) obtained from fitting the simulated spectra to the radio/sub-millimeter data. The forward slanted lines "III" show the regions in the parameter space that are excluded because the predicted infrared flux lies outside of the observed limits. Finally, the backward slanted lines "\\\" show the constraints on the image size at $1.3 \mathrm{~mm}$. The best-fit set of parameters for each simulation is obtained and is shown as a white circle or a white cross. The white circles are for fits with $\chi^{2}<1.5$, which we consider to be good fits; the white crosses are for $\chi^{2}>1.5$. Note that for the aOSANE models and the constant temperature aOMAD model, no set of parameters satisfies all of the observed constraints.

comparable for all simulations (see top panel of Figure 4 in Sagdowski et al. 2013a), the density normalizations shown here indicate that aOSANE has the highest physical accretion rate, while a9MAD has the lowest.

Fixing the electron temperature in the disk using the millimeter-to-centimeter spectrum. For each value of the electron-to-ion temperature ratio in the disk, the observed quiescent flux in the X-rays sets the electron density scale. Given that we have also fixed the values of two parameters, i.e., $i=60^{\circ}$ and $\beta_{\text {threshold }}=0.2$, the predicted millimeter-to-centimeter spectrum of $\mathrm{Sgr}^{*}$ * is now only a function of the remaining two parameters, which are the electron-to-ion temperature ratio in the disk, $\theta_{\text {disk}}$, and either the constant electron density in the funnel $T_{\mathrm{e} \text {,funnel }}$ or the electron-to-ion temperature ratio in the funnel $\theta_{\text {funnel }}$, depending on which plasma model we are considering. For each GRMHD simulation and for each plasma model, we show contours of $\chi^{2}$ values obtained by comparing the model prediction to the observed millimeter-to-centimeter spectrum of Sgr A*. In each panel, dark blue/purple colors represent small $\chi^{2}$ values (see the color bar) and hence are better fits.

Ruling out bright funnels using the infrared flux. In Figure 2, we showed that the predicted flux at infrared wavelengths is sensitive to the thermodynamic properties of the electrons in the funnel, with a weaker dependence on the electron temperature in the disk. In particular, when the electron temperature or the electron-to-ion temperature ratio in the funnel become large (and the funnel becomes very bright), the predicted infrared flux becomes too large to account for the range of observed infrared fluxes from Sgr $\mathrm{A}^{*}$. The same is true for the electron-to-ion temperature in the disk. In Figure 8, we use forward slanted lines " $/ / /$ " to show the regions of the parameter space that generate infrared fluxes outside of the observed range.
Table 2

The Best-fit Parameters for Different Simulations

\begin{tabular}{lcccccc}
\hline \hline Model & $\begin{array}{c}n_{\mathrm{e}} \\
\left(\theta_{\text {disk }}\right)\end{array}$ & $\theta_{\text {disk }}$ & $T_{\mathrm{e}, \text { funnel }}$ & $\theta_{\text {funnel }}$ & $\chi^{2}$ & \\
& & & & & \\
\hline a7SANE & $6.885 \times 10^{7}$ & 0.02371 & 56.23 & $\ldots$ & 1.178 & $\circ$ \\
a7SANE & $6.940 \times 10^{7}$ & 0.04217 & $\ldots$ & 0.17783 & 2.577 & $\times$ \\
a9SANE & $5.465 \times 10^{7}$ & 0.01000 & 31.62 & $\ldots$ & 0.674 & $\circ$ \\
a9SANE & $5.561 \times 10^{7}$ & 0.01778 & $\ldots$ & 0.05623 & 1.829 & $\times$ \\
aOMAD & $5.932 \times 10^{8}$ & 0.00056 & $\ldots$ & 0.01000 & 1.202 & $\circ$ \\
a7MAD & $2.495 \times 10^{8}$ & 0.00056 & 0.32 & $\ldots$ & 1.721 & $\times$ \\
a7MAD & $2.495 \times 10^{8}$ & 0.00056 & $\ldots$ & 0.00100 & 1.791 & $\times$ \\
a9MAD & $1.599 \times 10^{8}$ & 0.00075 & 1.78 & $\ldots$ & 1.471 & $\circ$ \\
a9MAD & $1.599 \times 10^{8}$ & 0.00075 & $\ldots$ & 0.00316 & 0.922 & $\circ$ \\
\hline
\end{tabular}

Notes. The above nine fits have a $\chi^{2}$ range from $\sim 0.5$ to $\sim 2.5$. The five fits with $\chi^{2}<1.5$ are equally good matches to the observational data of Sgr A*, which correspond to the white circles in Figure 8. The four fits with $\chi^{2}>1.5$, correspond to the white crosses in Figure 8, match the observation less well. The remaining three configurations, namely, aOSANE with the two different plasma models and AOMAD with constant temperature model, do not contain any set of parameters that can simultaneously satisfy all the observational constraints.

Rejecting models using the $1.3 \mathrm{~mm}$ image sizes. The final constraint on the model parameters arises from the comparison of the predicted to the observed $1.3 \mathrm{~mm}$ image sizes. We use backward slanted lines " $\backslash \backslash$ " to indicate the parameter space that is excluded by this constraint. With this final constraint folded in, we find the best-fit set of parameters for each simulation within the allowed regions of the parameter space and mark them with white circles or crosses in Figure 8 and list them in Table 2. The white circles in Figure 8 are for fits with $\chi^{2}<1.5$, which we consider to be good fits; the white crosses are for $\chi^{2}>1.5$. Note that for the aOSANE models and the constant temperature 


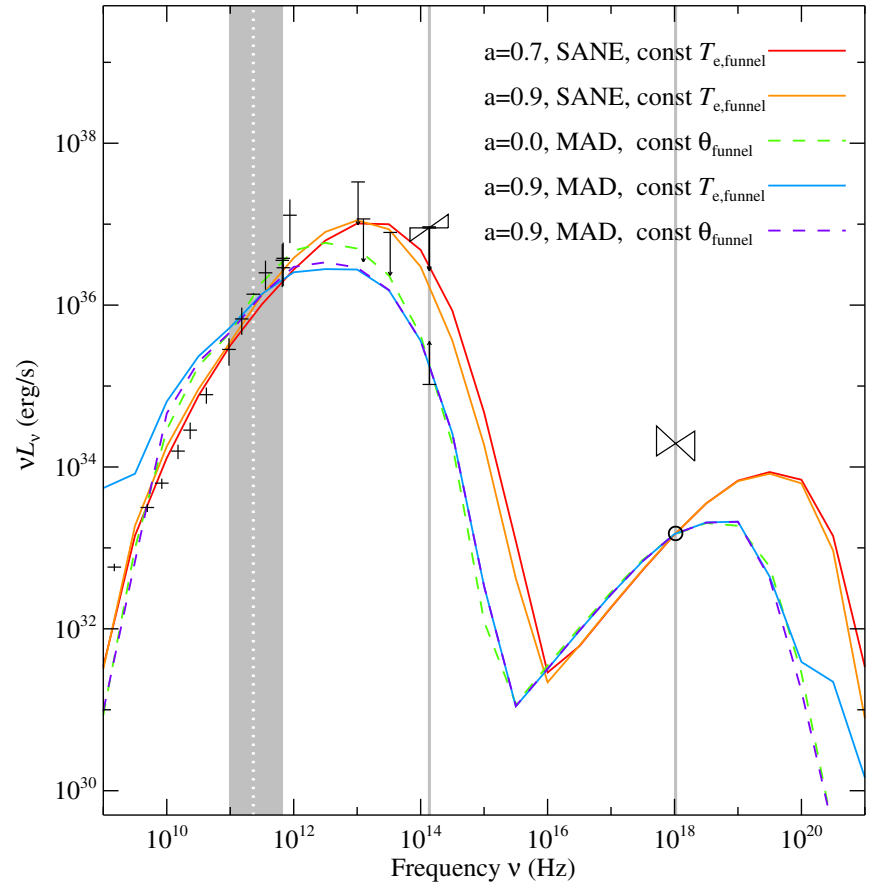

Figure 9. Five best-fit modeled spectra and the broadband spectral data that we used. The gray band between $v \approx 10^{11} \mathrm{~Hz}$ and $\approx 10^{12} \mathrm{~Hz}$ marks the frequency range over which we perform the least-squares fits. The gray line at $v \approx 10^{14} \mathrm{~Hz}$ marks the infrared frequency at which we used the range of fluxes observed at different times to impose an upper and lower bound on the models. The gray line at $v \approx 10^{18} \mathrm{~Hz}$ marks the X-ray frequency, at where we used $10 \%$ of the observed quiescent flux (the open circle below the bow-tie) to fix the density normalization in the flow. The white dotted line inside the gray band marks $\lambda=1.3 \mathrm{~mm}$, where we used the EHT measurement of the image size. The model parameters are marked as white circles in Figure 8 and listed in Table 2.

aOMAD temperature model, no set of parameters satisfies all of the observed constraints.

In Figure 9, we plot the best-fit model spectra together with the broadband spectral data that we use for the fitting. The gray band between $v \approx 10^{11} \mathrm{~Hz}$ and $\approx 10^{12} \mathrm{~Hz}$ marks the frequency range over which we perform the least-squares fits. The gray line at $v \approx 10^{14} \mathrm{~Hz}$ marks the infrared frequency at which we use the range of fluxes observed at different times to impose an upper and lower bound on the models. The gray line at $v \approx 10^{18} \mathrm{~Hz}$ marks the $\mathrm{X}$-ray frequency where we used $10 \%$ of the observed quiescent flux (the open circle below the bow-tie) to fix the density normalization in the flow. The white dotted line inside the gray band marks $\lambda=1.3 \mathrm{~mm}$, where we use the EHT measurement of the image size. The five curves correspond to the best-fit models with parameters marked as white circles in Figure 8 and listed in Table 2. For these fits, the two SANE simulations have spectral peaks near $v \approx 10^{13} \mathrm{~Hz}$ with $v L_{v} \approx 10^{37} \mathrm{erg} \mathrm{s}^{-1}$, and are indistinguishable from each other. The remaining three MAD simulations have spectral peaks near $v \approx 3 \times 10^{12} \mathrm{~Hz}$ with half an order of magnitude lower fluxes at these frequencies.

As a final consistency check, we plot in Figure 10 the range of image sizes for the best-fit models as functions of the observer's inclination. As discussed in Psaltis et al. (2014), in models that are dominated by disk emission, the inclination affects primarily the size of the $1.3 \mathrm{~mm}$ image, because of the Doppler effect. This is clearly seen in the first two panels that correspond to the SANE simulations. However, the dependence of the $1.3 \mathrm{~mm}$ image size on inclination for the aOMAD simulation is very weak, with a size that actually increases with inclination. In all cases, the value of $i=60^{\circ}$ that we have adopted for the inclination of the observer is consistent with observations.

\section{CONCLUSIONS}

In this paper, we analyzed the predicted spectra and images of various GRMHD simulations and plasma models for the accretion flow around Sgr A*, and narrowed the model parameter range by imposing the requirement that our predictions be consistent with the observed spectra and the $1.3 \mathrm{~mm}$ image size of Sgr A*. As seen in Figure 9, the best-fit model spectra agree with observational data very well. The models are most variable at infrared wavelengths, which is also consistent with observations. It is important to emphasize here that, if the spectral properties of Sgr A* were considered without any image size constraints, the range of allowed model parameter would be significantly wider. This demonstrates the power of using images with horizon-scale resolution to distinguish between models that would otherwise make seemingly similar predictions.

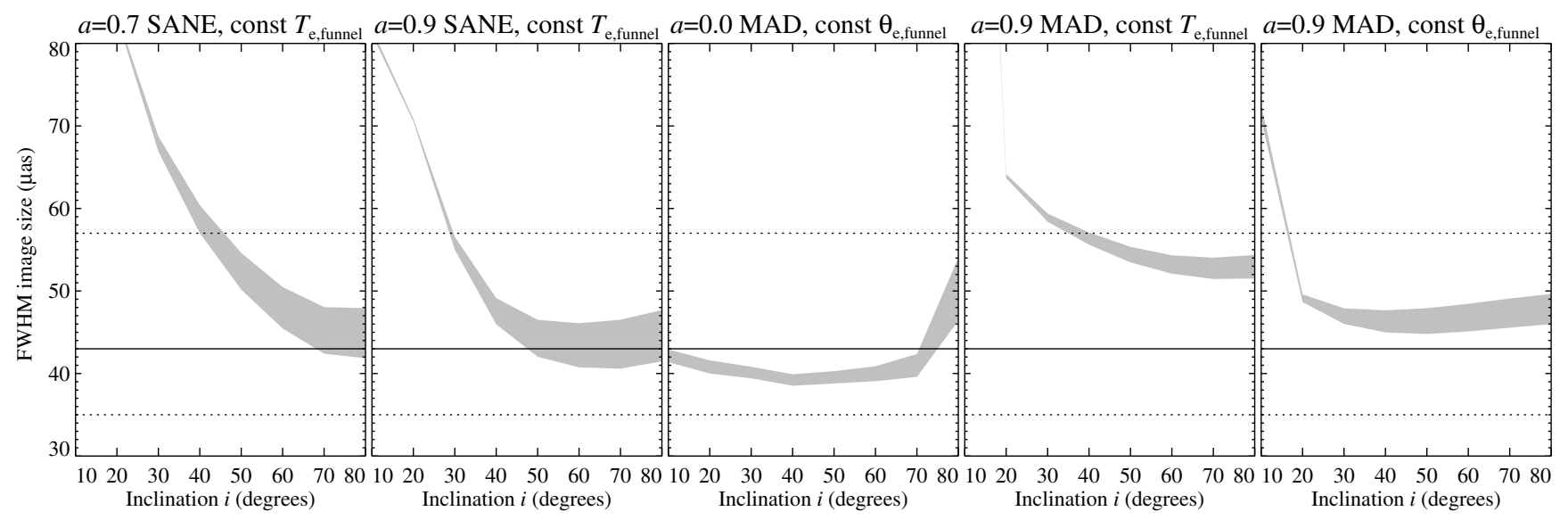

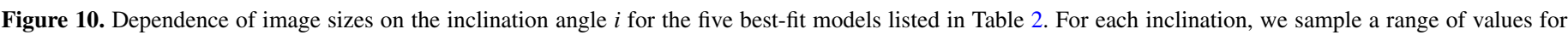

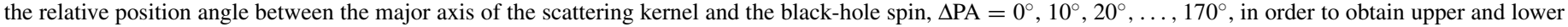

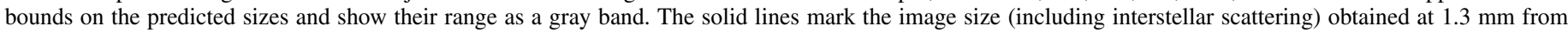

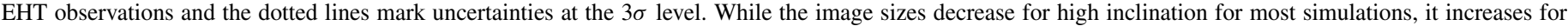

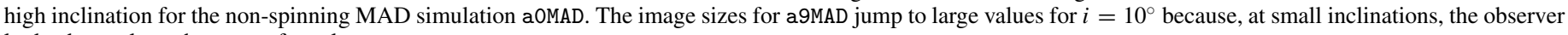
looks down along the strong funnel. 


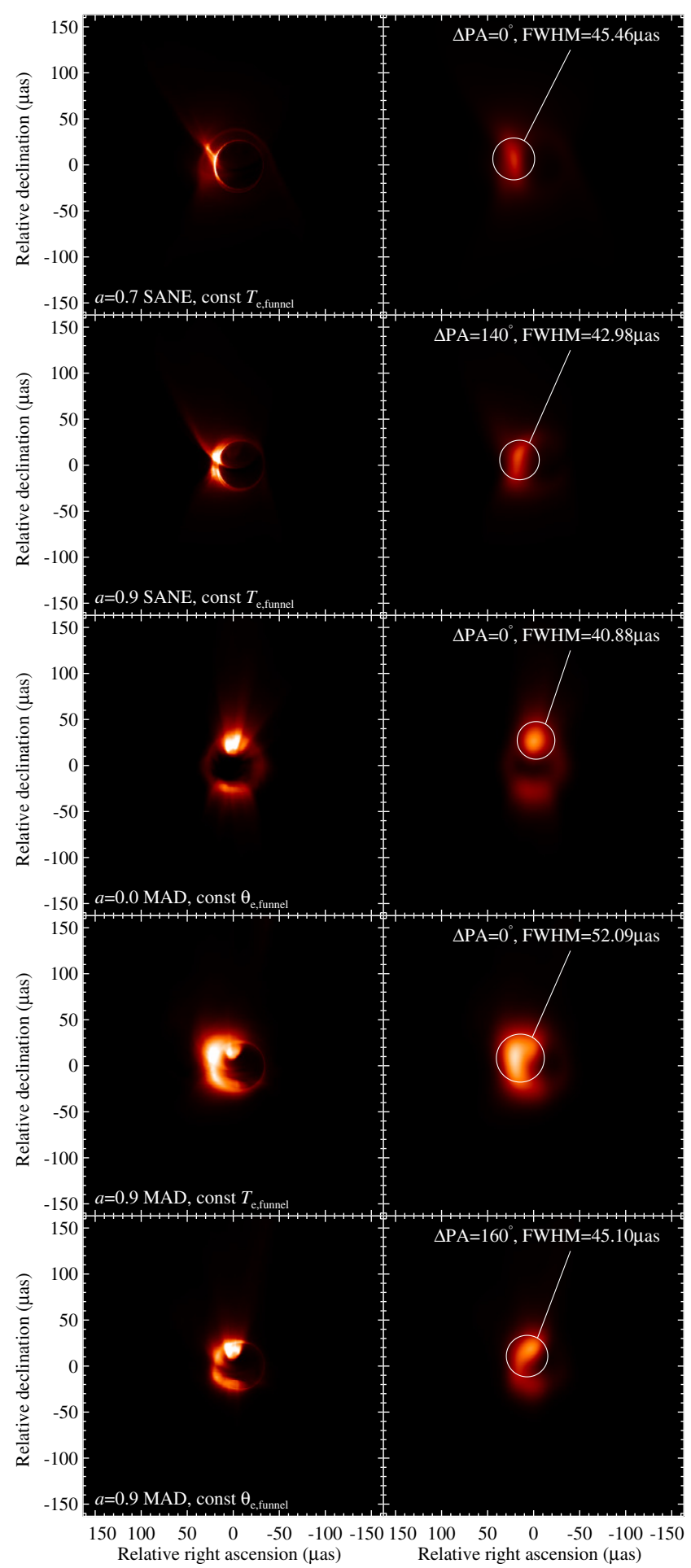

Figure 11. Predicted EHT images at $\lambda=1.3 \mathrm{~mm}$, for the five best-fit models shown with white circles in Figure 8 . The left column shows the results of the direct ray-tracing simulations, while the right column shows the scatterbroadened images. In the disk-dominated SANE simulations, the images have the characteristic crescent shape of a Doppler boosted accretion flow and a clear imprint of the black-hole shadow. In the jet-dominated MAD simulations, the image is formed primarily by the emission in the jet footprints but the shadow is still visible.

Future imaging observations from the completed EHT is expected to more easily distinguish disk-dominated from all funnel-dominated models. In Figure 11, we show the predicted $1.3 \mathrm{~mm}$ images for $\mathrm{Sgr}^{*}$ * for the GRMHD simulations and plasma model parameters that are consistent with all current spectra and imaging observations. With the completed EHT, it will be straightforward to distinguish between the first two pairs of images from the remaining three pairs of images shown, i.e., the disk-dominated from the funnel models, respectively. However, distinguishing among the various disk-dominated models and measuring the parameters of the black hole and of the plasma, will require additional information from the EHT, including the polarization and scale-dependent variability. We will explore these aspects of our models in future work.

This research is conducted at the University of Arizona and is supported by NSF grant AST 1312034. The Arizona team and R. N. acknowledge the support of NASA/NSF TCAN awards NNX14AB48G and NNX14AB47G, respectively. All ray tracing calculations were performed in the El Gato GPU cluster that is funded by NSF award 1228509. We thank Alexander Tchekhovskoy for providing the original derivations of the coordinate transformation used in the HARM simulations. We also thank Eliot Quataert and Jason Dexter for numerous discussions. F. Ö. thanks the Miller Institute for Basic Research in Science at the University of California Berkeley for their support and hospitality.

\section{REFERENCES}

Abramowicz, M. A., \& Fragile, P. C. 2013, LRR, 16, 1

Bower, G. C., Goss, W. M., Falcke, H., Backer, D. C., \& Lithwick, Y. 2006, ApJL, 648, L127

Bower, G. C., Markoff, S., Brunthaler, A., et al. 2014, ApJ, 790, 1

Broderick, A. E., Fish, V. L., Doeleman, S. S., \& Loeb, A. 2009, ApJ, 697, 45 Broderick, A. E., Fish, V. L., Doeleman, S. S., \& Loeb, A. 2011a, ApJ, 735, 110 Broderick, A. E., Loeb, A., \& Reid, M. J. 2011b, ApJ, 735, 57 Chan, C.-K., Liu, S., Fryer, C. L., et al. 2009, ApJ, 701, 521

Chan, C.-K., Psaltis, D., \& Özel, F. 2013, ApJ, 777, 13

Davis, S. W., Stone, J. M., \& Jiang, Y.-F. 2012, ApJS, 199, 9

Dexter, J., Agol, E., \& Fragile, P. C. 2009, ApJL, 703, L142

Dexter, J., Agol, E., Fragile, P. C., \& McKinney, J. C. 2010, ApJ, 717, 1092

Dexter, J., Agol, E., Fragile, P. C., \& McKinney, J. C. 2012, JPhCS, 372, 012023

Dexter, J., \& Fragile, P. C. 2011, ApJ, 730, 36

Dexter, J., \& Fragile, P. C. 2013, MNRAS, 432, 2252

Dodds-Eden, K., Gillessen, S., Fritz, T. K., et al. 2011, ApJ, 728, 37

Doeleman, S. S., Fish, V. L., Broderick, A. E., Loeb, A., \& Rogers, A. E. E. 2009, ApJ, 695, 59

Doeleman, S. S., Fish, V. L., Schenck, D. E., et al. 2012, Science, 338, 355

Doeleman, S. S., Weintroub, J., Rogers, A. E. E., et al. 2008, Nature, 455, 78

Falcke, H., \& Markoff, S. 2000, A\&A, 362, 113

Falcke, H., \& Markoff, S. B. 2013, CQGra, 30, 244003

Genzel, R., Schödel, R., Ott, T., et al. 2003, Nature, 425, 934

Ghez, A. M., Wright, S. A., Matthews, K., et al. 2004, ApJL, 601, L159

Gillessen, S., Eisenhauer, F., Trippe, S., et al. 2009, ApJ, 692, 1075

Leung, P. K., Gammie, C. F., \& Noble, S. C. 2011, ApJ, 737, 21

Mahadevan, R. 1998, Nature, 394, 651

McKinney, J. C., \& Gammie, C. F. 2004, ApJ, 611, 977

Mościbrodzka, M., \& Falcke, H. 2013, A\&A, 559, L3

Mościbrodzka, M., Falcke, H., Shiokawa, H., \& Gammie, C. F. 2014, A\&A, 570, A7

Mościbrodzka, M., Gammie, C. F., Dolence, J. C., Shiokawa, H., \& Leung, P. K. 2009, ApJ, 706, 497

Narayan, R., Mahadevan, R., Grindlay, J. E., Popham, R. G., \& Gammie, C. 1998, ApJ, 492, 554

Narayan, R., Sa̧dowski, A., Penna, R. F., \& Kulkarni, A. K. 2012, MNRAS, 426, 3241

Narayan, R., \& Yi, I. 1994, ApJL, 428, L13

Narayan, R., \& Yi, I. 1995, ApJ, 452, 710

Narayan, R., Yi, I., \& Mahadevan, R. 1995, Natur, 374, 623

Neilsen, J., Nowak, M. A., Gammie, C., et al. 2013, ApJ, 774, 42

Novikov, I. D., \& Thorne, K. S. 1973, in Black Holes (Les Astres Occlus), ed. C. Dewitt \& B. S. Dewitt (Paris: Gordon and Breach), 343

Özel, F., Psaltis, D., \& Narayan, R. 2000, ApJ, 541, 234

Psaltis, D., Narayan, R., Fish, V. L., et al. 2014, arXiv:1409.5447

Quataert, E. 2004, ApJ, 613, 322

Quataert, E., \& Gruzinov, A. 1999, ApJ, 520, 248 
Riquelme, M. A., Quataert, E., Sharma, P., \& Spitkovsky, A. 2012, ApJ, 755,50

Rybicki, G. B., \& Lightman, A. P. 1979, Radiative Processes in Astrophysics (New York: Wiley-Interscience)

Sądowski, A., Narayan, R., Penna, R., \& Zhu, Y. 2013a, MNRAS, 436, 3856

Sądowski, A., Sironi, L., Abarca, D., et al. 2013b, MNRAS, 432, 478

Shapiro, S. L., Lightman, A. P., \& Eardley, D. M. 1976, ApJ, 204, 187
Sharma, P., Quataert, E., \& Stone, J. M. 2008, MNRAS, 389, 1815

Shcherbakov, R. V., \& Baganoff, F. K. 2010, ApJ, 716, 504

Shcherbakov, R. V., Penna, R. F., \& McKinney, J. C. 2012, ApJ, 755, 133

Tchekhovskoy, A., Narayan, R., \& McKinney, J. C. 2011, MNRAS, 418, L79

Younsi, Z., Wu, K., \& Fuerst, S. V. 2012, A\&A, 545, A13

Yuan, F., \& Narayan, R. 2014, ARA\&A, 52, 529 


\title{
ERRATUM: “THE POWER OF IMAGING: CONSTRAINING THE PLASMA PROPERTIES OF GRMHD SIMULATIONS USING EHT OBSERVATIONS OF SGR A*” (2015, ApJ, 799, 1)
}

\author{
Chi-Kwan Chan ${ }^{1}$, Dimitrios Psaltis ${ }^{1}$, Feryal Özel $^{1}$, Ramesh Narayan $^{2}$, and Aleksander Sagdowski ${ }^{3}$ \\ ${ }^{1}$ Steward Observatory and Department of Astronomy, University of Arizona, 933 N. Cherry Ave., Tucson, AZ 85721 \\ ${ }^{2}$ Institute for Theory and Computation, Harvard-Smithsonian Center for Astrophysics, 60 Garden Street, Cambridge, MA 02138 \\ ${ }^{3}$ MIT Kavli Institute for Astrophysics and Space Research, 77 Massachusetts Ave, Cambridge, MA 02139 \\ Received 2015 June 7; accepted 2015 June 10; published 2015 July 6
}

The vertical axis markings on Figure 9 of the original publication is now corrected here in Figure 1. The error simply affected the values shown in the axis and does not change the results in the paper.

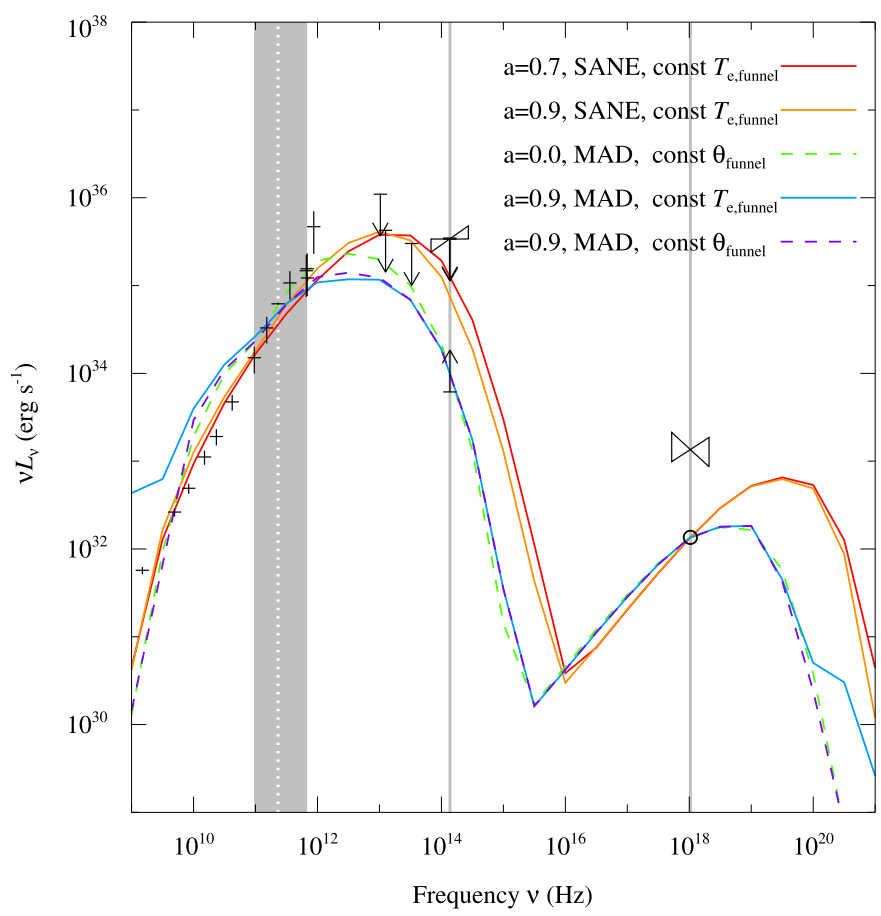

Figure 1. Updated Figure 9 from the original publication with corrected vertical axis markings: the five best-fit modeled spectra and the broadband spectral data that we used. The gray band between $\nu \approx 10^{11} \mathrm{~Hz}$ and $\approx 10^{12} \mathrm{~Hz}$ marks the frequency range over which we perform the least-squares fits. The gray line at $\nu \approx 10^{14} \mathrm{~Hz}$ marks the infrared frequency at which we used the range of fluxes observed at different times to impose an upper and lower bound on the models. The gray line at $\nu \approx 10^{18} \mathrm{~Hz}$ marks the X-ray frequency, where we used $10 \%$ of the observed quiescent flux (the open circle below the bow-tie) to fix the density normalization in the flow. The white dotted line inside the gray band marks $\lambda=1.3 \mathrm{~mm}$, where we used the EHT measurement of the image size. The model parameters are marked as white circles in Figure 8 and listed in Table 2 of the original text. 